\title{
Fair valuation of insurance liability cash-flow streams in continuous time: Theory
}

\author{
Łukasz Delong ${ }^{1}$, Jan Dhaene ${ }^{2 *}$, Karim Barigou ${ }^{2 *}$ \\ ${ }^{1}$ SGH Warsaw School of Economics \\ Collegium of Economic Analysis, Institute of Econometrics, \\ Niepodległości 162, Warsaw 02-554, Poland, \\ lukasz.delong@sgh.waw.pl \\ ${ }^{2} \mathrm{KU}$ Leuven \\ Actuarial Research Group, AFI, Faculty of Business and Economics, \\ Naamsestraat 69, 3000 Leuven, Belgium, \\ jan.dhaene@kuleuven.be \\ karim.barigou@kuleuven.be
}

*Jan Dhaene and Karim Barigou acknowledge the financial support of the Onderzoeksfonds KU Leuven (GOA/13/002). Karim Barigou is a $\mathrm{PhD}$ fellow of the Research Foundation - Flanders (FWO) [grant number 1146118N]. 


\begin{abstract}
We investigate fair (market-consistent and actuarial) valuation of insurance liability cash-flow streams in continuous time. We first consider one-period hedge-based valuations, where in the first step, an optimal dynamic hedge for the liability is set up, based on the assets traded in the market and a quadratic hedging objective, while in the second step, the remaining part of the claim is valuated via an actuarial valuation. Then, we extend this approach to a multi-period setting by backward iterations for a given discrete-time step $h$, and consider the continuous-time limit for $h \rightarrow 0$. We formally derive a partial differential equation for the valuation operator which satisfies the continuous-time limit of the multi-period, discrete-time iterations and prove that this valuation operator is actuarial and marketconsistent. We show that our continuous-time fair valuation operator has a natural decomposition into the best estimate of the liability and a risk margin. The dynamic hedging strategy associated with the continuous-time fair valuation operator is also established. Finally, the valuation operator and the hedging strategy allow us to study the dynamics of the net asset value of the insurer.
\end{abstract}

Keywords: Optimal quadratic hedging, actuarial valuation, marketconsistent valuation, fair valuation, partial differential equation, best estimate, risk margin, net asset value. 


\section{Introduction}

During the past decades, major changes have taken place in the way insurance liabilities are valuated. This is due to the emergence of solvency regimes such as Swiss Solvency Test, Solvency II and C-ROSS (Chinese solvency regulation) which are built on the paradigm that valuations should be risk-based and take into account available information provided by financial markets. One of the key changes has been the requirement of determining market-consistent values for insurance liabilities in order to guarantee a better matching between assets and liabilities (see e.g. Albrecher et al. (2018)).

Standard actuarial valuation is typically based on a diversification argument which justifies applying the law of large numbers among independent policyholders who face i.i.d. risks. This valuation is performed under the real-world measure $\mathbb{P}$ and defined as the expectation plus an additional risk margin to cover any not fully diversified and non-diversifiable risk. Based on historical data, the actuarial valuation involves a subjective judgement concerning the choice of the model and its parameters. For a survey of the classical insurance approach, we refer to Kaas et al. (2008) for non-life and Norberg (2014) for life insurance.

Risk-neutral valuation is, on the other hand, market-driven, hence objective, and based on the idea of hedging and replication. By no-arbitrage arguments, prices of contingent claims can be expressed as expectations under a so-called risk-neutral measure $\mathbb{Q}$. This approach dates back to the seminal paper of Black \& Scholes (1973) and was generalized to broad classes of processes. For an overview, see Delbaen \& Schachermayer (2006).

A large branch of literature investigated valuations in a market-consistent setting, trying to extend the arbitrage-free pricing operators (initially defined in a complete market) to the general set of non-hedgeable claims. Several approaches were considered such as utility indifference pricing (Hodges \& Neuberger (1989)) or risk-minimization techniques (Föllmer \& Schweizer (1988), Černỳ \& Kallsen (2009) and Delong (2013)). The notion of marketconsistency has been recently formalized by several authors as an extension 
of the notion of cash-invariance to all hedgeable claims, see e.g. Malamud et al. (2008), Pelsser \& Stadje (2014) and Dhaene et al. (2017).

It is important to notice that actuarial and risk-neutral valuations do not contradict each other, but are two types of valuations applied to different situations (diversifiable risks versus traded risks). In an insurance context, in which risks are partially traded and diversifiable, building a valuation framework which combines both approaches is primordial.

In this paper we investigate fair valuation of insurance liability cash-flow streams in continuous time, starting from the work of Dhaene et al. (2017), Barigou \& Dhaene (2018) and Barigou et al. (2018) in discrete time. In line with these papers, we define a fair valuation as a valuation which is actuarial (mark-to-model for claims independent of financial market evolutions) and market-consistent (mark-to-market for any hedgeable part of a claim). We first consider the one-period hedge-based valuations introduced by Dhaene et al. (2017), where in the first step, an optimal dynamic hedge for the liability is set up, based on the assets traded in the market and a quadratic hedging objective, while in the second step, the remaining part of the claim is valuated via an actuarial valuation. Then, we extend this approach to a multi-period setting by backward iterations for a given discrete-time step $h$, and consider the continuous-time limit for $h \rightarrow 0$. We formally derive a partial differential equation (PDE) for the valuation operator which satisfies the continuoustime limit of the multi-period, discrete-time iterations and prove that this valuation operator is actuarial and market-consistent. We show that our continuous-time fair valuation operator has a natural decomposition into the best estimate of the liability and a risk margin. The dynamic hedging strategy associated with the continuous-time fair valuation operator is also established. Finally, the valuation operator and the hedging strategy allow us to study the dynamics of the net asset value of the insurer. We focus on the theory of fair valuation of insurance liability cash-flow streams in continuous time. Examples relevant for practice and detailed interpretations can be found in Delong et al. (2018). 
We note that Pelsser (2010), Pelsser \& Stadje (2014) and Pelsser \& Ghalehjooghi (2016) also consider continuous-time valuation of insurance liabilities, taking into account both actuarial and market-consistent considerations. However, all these papers investigate the valuation of a particular type of contingent claims at a fixed future time and do not study hedging strategies. We consider a general framework of insurance and financial cash-flows at random times up to a terminal time and derive an optimal, dynamic investment strategy for hedging the cash-flows. The one-period valuation operators proposed in this research are also different from the ones proposed in Pelsser (2010), Pelsser \& Stadje (2014) and Pelsser \& Ghalehjooghi (2016).

We should also mention the paper by Happ et al. (2015) who consider valuation of insurance liability cash-flow streams in a multi-period, discrete-time model. The best estimate of liability is obtained by sequential local risk minimization which describes the dynamically hedgeable part of the insurance cash-flows. These sequential risk minimization are obtained by orthogonal $L^{2}$ projections of the liability onto the space of hedgeable payoffs. Hence, the best estimate of the liability is the market value of the hedgeable pay-off derived from the orthogonal projection of the liability. The technical provision is then defined as the arbitrage-free price (including risk premiums for the non-hedgeable risks) of the insurance cash-flows. The multi-period valuation operator from Happ et al. (2015) has some similarities with our multi-period valuation operator, yet the constructions are different. Moreover, in this paper we focus on the continuous-time valuation operator and its properties.

This paper is organized as follows. Section 2 describes the financial and insurance model as well as the notions of market-consistent, actuarial and fair valuations and hedging strategies in continuous time. In Section 3, we present our one-period valuation problem and derive the optimal hedging strategy for the liability by quadratic hedging. Section 4 introduces the multiperiod valuation operator by iterating the one-period valuation operator. Section 5 investigates the continuous-time limit of the multi-period valuation operator with quadratic one-period actuarial valuation operators (standard 
deviation and variance risk margins) used for valuating the non-hedgeable part of the liability. Section 6 discusses extensions beyond quadratic oneperiod actuarial valuations. All proofs are included in Section 7.

\section{Financial and Insurance Model}

Throughout the paper, we work on a probability space $(\Omega, \mathbb{F}, \mathbb{P})$ equipped with a filtration $\mathbb{F}=\left(\mathcal{F}_{t}\right)_{0 \leq t \leq T}$ and a finite time horizon $T<\infty$. On the probability space $(\Omega, \mathbb{F}, \mathbb{P})$ we define a standard two-dimensional Brownian motion $\left(W_{1}, W_{2}\right)=\left(W_{1}(t), W_{2}(t), 0 \leq t \leq T\right)$ and a càdlàg (rightcontinuous with left limits) counting process $N=(N(t), 0 \leq t \leq T)$. The Brownian motions $\left(W_{1}, W_{2}\right)$ are used to model the financial risk and the $\sigma$-algebra $\mathcal{F}_{t}^{W_{1}, W_{2}}=\sigma\left(W_{1}(u), W_{2}(u), u \in[0, t]\right)$ contains all information on the evolution of the financial assets up to and including time $t$. The counting process $N$ is used to model the insurance risk and the $\sigma$-algebra $\mathcal{F}_{t}^{N}=\sigma(N(u), u \in[0, t])$ contains information on the number of in-force policies in the insurance portfolio up to and including time $t$. The insurance risk is not traded in the market, and the financial risk contains a tradeable and a non-tradeable component modelled with $W_{1}$ and $W_{2}$, respectively. We assume:

(A1) The subfiltrations $\mathbb{F}^{W_{1}, W_{2}}=\left(\mathcal{F}_{t}^{W_{1}, W_{2}}\right)_{0 \leq t \leq T}$ and $\mathbb{F}^{N}=\left(\mathcal{F}_{t}^{N}\right)_{0 \leq t \leq T}$ are independent, and we set $\mathbb{F}=\mathbb{F}^{W_{1}, W_{2}} \times \mathbb{F}^{N}$.

Under assumption (A1) the financial risk is independent of the insurance risk.

\subsection{The financial market}

The financial market consists of a risk-free asset $R=(R(t), 0 \leq t \leq T)$ and two risky assets: $Y=(Y(t), 0 \leq t \leq T)$ and $F=(F(t), 0 \leq t \leq T)$. The 
price of the risk-free asset grows exponentially:

$$
\frac{d R(t)}{R(t)}=r d t, \quad 0 \leq t \leq T, \quad R(0)=1
$$

We assume that the prices of the risky assets $Y$ and $F$ are modelled with correlated geometric Brownian motions and follow the dynamics

$$
\begin{aligned}
& \frac{d Y(t)}{Y(t)}=\mu_{Y} d t+\sigma_{Y} d W_{Y}(t), \quad 0 \leq t \leq T, \quad Y(0)=y_{0}, \\
& \frac{d F(t)}{F(t)}=\mu_{F} d t+\sigma_{F} d W_{F}(t), \quad 0 \leq t \leq T, \quad F(0)=f_{0},
\end{aligned}
$$

where $\mu_{Y}, \mu_{F}, \sigma_{Y}, \sigma_{F}$ are non-negative real numbers denoting the drifts and volatilities of the risky assets, respectively, while $\left(W_{Y}, W_{F}\right)$ denotes a correlated two-dimensional Brownian motion with correlation coefficient $\rho$. We define

$$
W_{Y}(t)=W_{1}(t), \quad W_{F}(t)=\rho W_{1}(t)+\sqrt{1-\rho^{2}} W_{2}(t), \quad 0 \leq t \leq T .
$$

where $\left(W_{1}, W_{2}\right)$ is the standard two-dimensional Brownian motion on $(\Omega, \mathbb{F}, \mathbb{P})$, i.e. $W_{1}$ is independent of $W_{2}$. We will use both $\left(W_{Y}, W_{F}\right)$ and $\left(W_{1}, W_{2}\right)$ in the sequel.

The insurance company can invest in the risk-free asset $R$ and in the risky asset $Y$. The risky asset $F$ is not available for trading. The risky asset $F$ is the underlying investment fund for the contracts sold by the insurance company.

\subsection{The insurance portfolio}

The insurance company holds a homogeneous portfolio which consists of $n$ identical insurance policies. All policyholders have the same age (or are classified into the same age group) and are entitled to three types of benefits: a continuous annuity benefit $A$ paid in $[0, T]$ as long as the insured is alive, a death benefit $D$ paid at the moment that the insured dies (provided he dies in $[0, T])$ and a survival benefit $S$ paid at terminal time $T$ if the insured survives 
that time. The benefits $A, D$ and $S$ are allowed to be time-dependent and contingent on the values of the risky assets $(Y, F)$.

The process $N$ counts the number of deaths in the insurance portfolio. We assume:

(A2) The lifetimes of the policyholders $\left(\tau_{k}\right)_{k=1, \ldots, n}$ at policy inception are independent and identically exponentially distributed, i.e.

$$
\mathbb{P}\left(\tau_{k}>t\right)=e^{-\int_{0}^{t} \lambda(s) d s}, \quad k=1, \ldots, n, \quad 0 \leq t \leq T .
$$

The function $\lambda:[0, T] \mapsto(0, \infty)$ is continuously differentiable, i.e. $\lambda \in C^{1}([0, T])$, and strictly positive.

We have that

$$
N(t)=\sum_{k=1}^{n} \mathbf{1}\left\{\tau_{k} \leq t\right\}, \quad 0 \leq t \leq T
$$

The deterministic function $\lambda$ describes the mortality intensity of the policyholders in the insurance portfolio. We introduce the compensated counting process $\tilde{N}=(\tilde{N}(t), 0 \leq t \leq T)$ :

$$
\tilde{N}(t)=N(t)-\int_{0}^{t}(n-N(s-)) \lambda(s) d s, \quad 0 \leq t \leq T,
$$

which is an $\mathbb{F}$-martingale. The compensated counting process will be used to construct the stochastic integral describing the non-hedgeable insurance risk. We also introduce the process $J=(J(t), 0 \leq t \leq T)$ :

$$
J(t)=n-N(t), \quad 0 \leq t \leq T
$$

which counts the number of in-force policies in the insurance portfolio.

The cash-flow stream of the benefit payments of the portfolio is modelled by the process $B=(B(t), 0 \leq t \leq T)$. This process is described by the following equation

$$
\begin{aligned}
B(t)= & \int_{0}^{t}(n-N(u-)) A(u, Y(u), F(u)) d u+\int_{0}^{t} D(u, Y(u), F(u)) d N(u) \\
& +(n-N(T)) S(Y(T), F(T)) \mathbf{1}_{t=T}, \quad 0 \leq t \leq T
\end{aligned}
$$


The process $B$ is $\mathbb{F}$-adapted. The general benefit stream (2.5) was considered in Møller \& Steffensen (2007) who studied market-consistent valuation methods in life insurance. Special cases of (2.5), and their fair values, are investigated in details in Delong et al. (2018).

We assume:

(A3) The functions $A:[0, T] \times(0, \infty) \times(0, \infty) \mapsto[0, \infty), D:[0, T] \times(0, \infty) \times$ $(0, \infty) \mapsto[0, \infty)$ and $S:(0, \infty) \times(0, \infty) \mapsto[0, \infty)$ are Lipschitz continuous and have linear growth in $(y, f)$ :

$$
\begin{aligned}
\left|A\left(t, y_{1}, f_{1}\right)-A\left(t, y_{2}, f_{2}\right)\right| & \leq K\left(\left|y_{1}-y_{2}\right|+\left|f_{1}-f_{2}\right|\right), \\
|A(t, y, f)| & \leq K(1+|y|+|f|), \\
\left|D\left(t, y_{1}, f_{1}\right)-D\left(t, y_{2}, f_{2}\right)\right| & \leq K\left(\left|y_{1}-y_{2}\right|+\left|f_{1}-f_{2}\right|\right), \\
|D(t, y, f)| & \leq K(1+|y|+|f|), \\
\left|S\left(y_{1}, f_{1}\right)-S\left(y_{2}, f_{2}\right)\right| & \leq K\left(\left|y_{1}-y_{2}\right|+\left|f_{1}-f_{2}\right|\right), \\
|S(y, f)| & \leq K(1+|y|+|f|) .
\end{aligned}
$$

Moreover, the functions $A$ and $D$ are continuous in $t$ and satisfy the conditions:

$$
\begin{aligned}
\left|A\left(t_{1}, y, f\right)-A\left(t_{2}, y, f\right)\right| & \leq K\left|t_{1}-t_{2}\right|(1+|y|+|f|), \\
\left|D\left(t_{1}, y, f\right)-D\left(t_{2}, y, f\right)\right| & \leq K\left|t_{1}-t_{2}\right|(1+|y|+|f|),
\end{aligned}
$$

\subsection{Fair and arbitrage-free valuations in the combined financial and insurance model}

By paying the benefits from the process $B$, we observe that the insurer is exposed to three sources of risk:

- Tradeable financial risk $Y$ : The fluctuations of the risky asset $Y$ impact the payment process 2.5). This risk can be perfectly hedged by trading in $Y$. 
- Non-tradeable financial risk $F$ : The variations of the risky asset $F$ impact the benefit stream 2.5 as well. This risk can be partially hedged by trading in $Y$, since $Y$ and $F$ are correlated. The higher the absolute value of the correlation parameter, the better the hedge.

- Non-tradeable insurance risk $N$ : The non-tradeable insurance risk arises since the policyholders die at random times and the death-related benefits have to be paid at unpredictable times. This risk cannot be hedged since it is assumed to be independent of the financial market.

Our goal is to attach a fair value at any time $t$ in $[0, T]$ to the future claims from the benefit stream $B$ and to find the dynamic hedging strategy which underlies the fair value.

In the sequel we will use the notions of market-consistent, actuarial and fair valuations and hedging strategies introduced by Dhaene et al. (2017) and further studied by Barigou \& Dhaene (2018) and Barigou et al. (2018) in a multi-period, discrete time setting. Hereafter, we adapt these notions to our continuous-time model. To do so, we consider two particular cases of the benefit stream $B$. First, we consider the case in which the benefits are only contingent on the tradeable financial risk $Y$ :

$$
B^{Y}(t)=\int_{0}^{t} A(u, Y(u)) d u+S(Y(T)) \mathbf{1}_{t=T}, \quad 0 \leq t \leq T .
$$

Secondly, we consider the case in which the benefits are only contingent on the non-tradeable insurance risk $N$ :

$$
\begin{aligned}
B^{N}(t)= & \int_{0}^{t}(n-N(u-)) A(u) d u+\int_{0}^{t} D(u) d N(u) \\
& +(n-N(T)) S \mathbf{1}_{t=T}, \quad 0 \leq t \leq T .
\end{aligned}
$$

The process $B^{Y}$ can be perfectly replicated by trading in $Y$ and will be called a hedgeable process. The process $B^{N}$ is independent of the financial risks $(Y, F)$ and will be called an orthogonal process. Clearly, the process $B^{Y}$ is $\mathbb{F}^{W_{1}, W_{2}}$-adapted and the process $B^{N}$ is $\mathbb{F}^{N}$-adapted. 
Definition 2.1. Let $\varphi_{B(t, T]}(t)$ denote the value at time $t \in[0, T]$ of the future claims from the process $B$. For each $t \in[0, T]$, the operator $\varphi_{B(t, T]}(t)$ maps a $\sigma\left(\mathbf{1}\{u \geq t\} W_{1}(u), \mathbf{1}\{u \geq t\} W_{2}(u), u \in[0, T]\right) \times \sigma(\mathbf{1}\{u \geq t\} N(u), u \in[0, T])-$ measurable random variable into an $\mathcal{F}_{t}$-measurable random variable. We will say that

- the valuation operator $\varphi$ is market-consistent if for any process $B$ and any hedgeable process $B^{Y}$, as defined in (2.6), we have that

$$
\begin{aligned}
\varphi_{B(t, T]+B^{Y}(t, T]}(t) & =\varphi_{B(t, T]}(t)+\varphi_{B^{Y}(t, T]}(t), \\
\text { with } & \\
\varphi_{B^{Y}(t, T]}(t) & =\mathbb{E}^{\hat{\mathbb{Q}}}\left[\int_{t}^{T} e^{-r(u-t)} d B^{Y}(u) \mid \mathcal{F}_{t}^{W_{1}}\right], 0 \leq t \leq T,
\end{aligned}
$$

where $\widehat{\mathbb{Q}}$ denotes the unique equivalent martingale measure for the traded risky asset $Y$,

- the valuation operator $\varphi$ is actuarial if for any orthogonal process $B^{N}$, as defined in (2.7), we have that

$$
\begin{aligned}
\varphi_{B^{N}(t, T]}(t)= & \mathbb{E}^{\mathbb{P}}\left[\int_{t}^{T} e^{-r(u-t)} d B^{N}(u) \mid \mathcal{F}_{t}^{N}\right] \\
& +R M_{B^{N}(t, T]}^{a c t}(t), \quad 0 \leq t \leq T,
\end{aligned}
$$

where, for each $t \in[0, T]$, the operator $R M_{B^{N}(t, T]}^{\text {act }}(t)$ maps a $\sigma(1\{u \geq$ t\} $N(u), u \in[0, T])$-measurable random variable into an $\mathcal{F}_{t}^{N}$-measurable random variable.

- the valuation operator $\varphi$ is fair if it is market-consistent and actuarial.

The definition of the valuation operator for the benefit stream as a measurable mapping is taken from Cheridito et al. (2006). The market-consistency condition (2.8) imposes that the claims which can be replicated by traded assets should be valuated at the price of their perfect hedge, since they do not carry any risk. The mark-to-model condition (2.9) corresponds to the 
traditional actuarial valuation of insurance liabilities. It postulates that any orthogonal process, which is independent of the financial risk, should be valuated by an operator which only takes into account the information about the insurance risk, and the value of an orthogonal process is determined by the expected discounted future benefits and a risk loading. Let us note that any valuation operator $\varphi$ can be decomposed into the conditional expected value, under the real-world measure $\mathbb{P}$, of the discounted benefits and another valuation operator $R M$ which we call a risk margin valuation operator. The risk margin valuation operator $R M$ is called actuarial if $R M$ reduces to an actuarial risk margin $R M^{a c t}$ as defined 2.9 when applied to an orthogonal benefit stream $B^{N}$. The definition of the risk margin $R M^{a c t}$ is very general. We can have some obvious examples:

$$
\begin{aligned}
& R M_{B^{N}(t, T]}^{a c t}(t)=S D^{\mathbb{P}}\left[\int_{t}^{T} e^{-r(u-t)} d B^{N}(u) \mid \mathcal{F}_{t}^{N}\right] \\
& R M_{B^{N}(t, T]}^{a c t}(t)=V a R_{1-\beta}^{\mathbb{P}}\left[\int_{t}^{T} e^{-r(u-t)} d B^{N}(u) \mid \mathcal{F}_{t}^{N}\right],
\end{aligned}
$$

as well as more sophisticated valuation operators:

$$
R M_{B^{N}(t, T]}^{a c t}(t)=\mathbb{E}^{\mathbb{P}}\left[\int_{t}^{T} e^{-r(u-t)} g(u, N(u)) d u \mid \mathcal{F}_{t}^{N}\right]
$$

where $g$ is a deterministic function used to quantify the local risk of the benefit stream $B^{N}$. Since the risk of $B^{N}$ is generated by the process $N$, the function $g$ only depends on $N$. The valuation operator (2.11) is an example of a so-called $g$-expectation and a dynamic risk measure, see Rosazza Gianin (2006).

After having considered desirable properties that a valuation operator should have, we introduce similar properties for the hedging operator.

Definition 2.2. Let $\left(\theta_{B(t, T]}(s), t \leq s \leq T\right)$ denote the hedging strategy in the risky asset $Y$ for the future claims from the process $B$ (the amount of money invested in the risky asset $Y$ ). We will say that 
- the strategy $\theta$ is market-consistent if for any process $B$ and any hedgeable process $B^{Y}$ we have that

$$
\theta_{B(t, T]+B^{Y}(t, T]}(s)=\theta_{B(t, T]}(s)+v_{y}(s, Y(s)) Y(s), \quad t \leq s \leq T,
$$

where $v(t, y)=\mathbb{E}^{\hat{\mathbb{Q}}}\left[\int_{t}^{T} e^{-r(u-t)} d B^{Y}(u) \mid Y(t)=y\right]$ and $\hat{\mathbb{Q}}$ denotes the unique equivalent martingale measure for the traded risky asset $Y$,

- the strategy $\theta$ is actuarial if for any orthogonal process $B^{N}$ we have that

$$
\theta_{B^{N}(t, T]}(s)=0, \quad t \leq s \leq T
$$

- the strategy $\theta$ is fair if it is market-consistent and actuarial.

The notions of market-consistent, actuarial and fair strategies were first introduced in Dhaene et al. (2017) in a single-period setting and further generalized in Barigou et al. (2018) in a dynamic discrete setting.

The market-consistency condition (2.12) imposes the natural condition that any hedgeable claim should be hedged by its replicating hedging strategy. Let us remark that the second term in 2.12 is the delta-hedging strategy for $B^{Y}$, which is the replicating strategy for $B^{Y}$. The condition 2.13 imposes that any orthogonal process is hedged with a risk-free investment, which seems reasonable since the orthogonal process is independent of the financial risks $(Y, F)$.

We now describe the equivalent martingale measures in our combined financial and insurance model and the dynamics of the risk factors under an equivalent martingale measure. Let us define

$$
\begin{aligned}
\frac{d \mathbb{Q}}{d \mathbb{P}} \mid \mathcal{F}_{t} & =\mathcal{M}^{\zeta, \chi}(t), \quad 0 \leq t \leq T, \\
\frac{d \mathcal{M}^{\zeta, \chi}(t)}{\mathcal{M}^{\zeta, \chi}(t-)} & =-\left(\frac{\mu_{Y}-r}{\sigma_{Y}}\right) d W_{1}(t)+\zeta(t) d W_{2}(t)+\chi(t) d \tilde{N}(t),
\end{aligned}
$$

where $(\zeta, \chi)$ are predictable, Markov processes with respect to the natural filtration $\mathbb{F}^{Y, F, N}=\mathbb{F}^{Y, F} \times \mathbb{F}^{N}$ generated by $(Y, F, N)$ and they satisfy the 
conditions:

$$
\begin{aligned}
& \sup _{t \in[0, T]} \mathbb{E}\left[\int_{t}^{T}|\zeta(s)|^{2} d s \mid \mathcal{F}_{t}^{Y, F, N}\right] \leq K \\
& -1+\epsilon \leq|\chi(t)| \leq K, \quad 0 \leq t \leq T, \epsilon>0
\end{aligned}
$$

By Lemma 1 in Morlais (2010) the process $\mathcal{M}^{\zeta, \chi}$ is a $\mathbb{P}$-martingale and can be used to define the equivalent probability measure. Moreover, from Theorem 3.1 in Kazamaki (2006) and boundedness of $\chi$ we conclude that $\mathbb{E}\left[\left|\mathcal{M}^{\zeta, \chi}(T)\right|^{q}\right]<\infty$ for some $q>1$. By Girsanov's theorem we deduce that

$$
\begin{aligned}
& W_{1}^{\mathbb{Q}}(t)=W_{1}(t)+\int_{0}^{t}\left(\frac{\mu_{Y}-r}{\sigma_{Y}}\right) d s, \quad 0 \leq t \leq T, \\
& W_{2}^{\mathbb{Q}}(t)=W_{2}(t)-\int_{0}^{t} \zeta(s) d s, \quad 0 \leq t \leq T,
\end{aligned}
$$

and

$$
\tilde{N}^{\mathbb{Q}}(t)=N(t)-\int_{0}^{t}(n-N(s-)) \lambda(s)(1+\chi(s)) d s, \quad 0 \leq t \leq T,(
$$

are $\mathbb{Q}$-Brownian motions and a $\mathbb{Q}$-compensated counting process, respectively. By 2.2)-2.4 we also have the following dynamics for the risky assets $(Y, F)$ :

$$
\begin{aligned}
\frac{d Y(t)}{Y(t)}= & r d t+\sigma_{Y} d W_{Y}^{\mathbb{Q}}(t), \quad 0 \leq t \leq T, \\
\frac{d F(t)}{F(t)}= & \left(\mu_{F}-\frac{\mu_{Y}-r}{\sigma_{Y}} \sigma_{F} \rho\right. \\
& \left.+\sigma_{F} \sqrt{1-\rho^{2}} \zeta(t)\right) d t+\sigma_{F} d W_{F}^{\mathbb{Q}}(t), \quad 0 \leq t \leq T,
\end{aligned}
$$

where $W_{Y}^{\mathbb{Q}}, W_{F}^{\mathbb{Q}}$ are correlated with correlation coefficient $\rho$.

Arbitrage-free pricing theory tells us that the claims from the process $B$ should be priced by

$$
\varphi_{B(t, T]}(t)=\mathbb{E}^{\mathbb{Q}}\left[\int_{t}^{T} e^{-r(s-t)} d B(s) \mid \mathcal{F}_{t}\right], \quad 0 \leq t \leq T,
$$

for some equivalent martingale measure $\mathbb{Q}$. Specifying the equivalent martingale measure $\mathbb{Q}$ for pricing $B$ is equivalent to specifying the risk premiums 
$(\zeta, \chi)$ for the non-tradeable financial and insurance risks. Let us recall that in our model the non-tradeable financial risk $F$ is correlated with the tradeable financial risk $Y$. The part of the non-tradeable financial risk, which is correlated with the tradeable financial risk, is priced with the risk premium $\frac{\mu_{Y}-r}{\sigma_{Y}} \sigma_{F} \rho$. The independent part of the non-tradeable financial risk is priced with the risk premium $\zeta$. In addition to the non-tradeable financial risk, we have an independent non-tradeable insurance risk. Under an equivalent martingale measure $\mathbb{Q}$ the counting process $N$ with intensity $\lambda(t)$ becomes a counting process with a new intensity $(1+\chi(t)) \lambda(t)$, where $\chi$ denotes a risk premium for the independent non-tradeable insurance risk. Since the processes $(\zeta, \chi)$ are used to price the non-tradeable risks, their values are subjective and related to the pricing mechanism applied by the investor and his risk aversion preferences.

For pricing the general benefit stream $B$ we can choose $\mathbb{F}^{Y, F, N}$-predictable processes $(\zeta, \chi)$. E.g. if the insurer offers a death guarantee which value depends on $(Y, F)$, then it may make sense to assume that the risk premium for the insurance risk depends on the risky assets $(Y, F)$ - a deep in-the-money death guarantee could potentially increase the mortality intensity under the pricing measure. However, when we price an orthogonal process $B^{N}$, then measurability restrictions on $(\zeta, \chi)$ are natural. We assume:

(A4) If the equivalent martingale measure (2.14) is used for pricing the orthogonal benefit stream $B^{N}$, then $\zeta=0$ and $\chi$ should be an $\mathbb{F}^{N}$ predictable, Markov process.

Finally, let us consider a very special case of $\mathbb{Q}$. Let us define

$$
\begin{aligned}
\frac{d \hat{\mathbb{Q}}}{d \mathbb{P}} \mid \mathcal{F}_{t} & =\hat{\mathcal{M}}(t), \quad 0 \leq t \leq T, \\
\frac{d \hat{\mathcal{M}}(t)}{\hat{\mathcal{M}}(t)} & =-\left(\frac{\mu_{Y}-r}{\sigma_{Y}}\right) d W_{1}(t) .
\end{aligned}
$$

Then $\hat{\mathbb{Q}}$ is the unique equivalent martingale measure for the traded risky asset $Y$ in the complete market consisting of $(R, Y)$. The dynamics of the 
counting process and the risky assets are in this case given by $(2.16)-(2.17)$ with $\zeta(t)=\chi(t)=0$.

\section{The one-period valuation operator and dy- namic hedging strategies}

Let us start with the benefit stream $B$ which only includes claims at the terminal time $T$. In this case, the benefit stream (2.5) takes the form

$$
B(t)=(n-N(T)) S(Y(T), F(T)) \mathbf{1}_{t=T}, \quad 0 \leq t \leq T .
$$

We define

$$
\triangle B(T)=(n-N(T)) S(Y(T), F(T))
$$

which specifies the benefits from the process $B$ to be paid at the terminal time $T$. We consider the one-period valuation operator $\varrho$ for the benefit stream $B$ :

$$
\varrho(B)=V_{B}(0)+\pi\left(\left(\triangle B(T)-V_{B}(T)\right) e^{-r T}\right),
$$

where $V_{B}(t)$ denotes the time- $t$ value of a hedging portfolio for the claims from the process $B$ (for $t=0, T$ ), and $\pi$ denotes a one-period actuarial valuation operator. The hedging portfolio $V_{B}$ could be e.g. derived by minimizing the mean-square hedging error for the terminal claims $\triangle B(T)$ under the real-world measure. The actuarial valuation $\pi$ is next applied to the discounted remaining non-hedgeable risks. The valuation operator (3.1) splits the valuation of the benefit stream $B$ into two parts: $V_{B}(0)$ gives the value of the hedgeable part of $B$ and $\pi$ gives the value of the non-hedgeable part of $B$.

The one-period valuation operator (3.1) was introduced by Dhaene et al. (2017), and next studied in a multi-period setting by Barigou \& Dhaene 
(2018) and Barigou et al. (2018). In particular, the multi-period iterations

$$
\begin{aligned}
\varphi_{\triangle B(T)}(T) & =\triangle B(T), \\
\varphi_{B(t, T]}(t) & =\varrho\left(\int_{t}^{t+h} d \tilde{B}(s)\right), \quad t=0, h, \ldots, T-h, \\
\tilde{B}(s) & =\varphi_{B(t+h, T]}(t+h) \mathbf{1}\{s=t+h\}, \quad t \leq s \leq t+h,
\end{aligned}
$$

were investigated in Barigou et al. (2018). The iterations (3.2) allow us to define the values of the future claims from the process $B$ at the discrete times $t=0, \ldots, T-h, T$.

In this paper we modify the approach from Dhaene et al. (2017), Barigou \& Dhaene (2018) and Barigou et al. (2018) as follows:

- We allow for annuity, death and survival benefits described by the process $B$ in 2.5 .

- We allow for dynamic trading in the financial market. This assumption is consistent with continuous-time financial models.

- We assume that the hedging strategy is predictable with respect to the filtration $\mathbb{F}=\mathbb{F}^{W_{1}, W_{2}} \times \mathbb{F}^{N}$ generated by the financial and insurance risks. This implies, in particular, that the new information on the financial and the insurance risk updates the hedging strategy.

- We change the optimization functional for deriving the one-period hedging portfolio so that the optimal, dynamic hedging strategy is fair.

- We take the limit $h \rightarrow 0$ in (3.2) and derive a PDE for the valuation operator which satisfies the continuous-time limit of the multi-period, discrete-time iterations (3.2) with quadratic one-period actuarial valuation operators.

- We deduce the dynamic hedging strategy which underlies our continuoustime valuation operator. 
- We discuss the structure of the derived PDE and we propose a PDE for a general continuous-time valuation operator in our continuous-time model with dynamic hedging.

As mentioned in the introduction, our research is also closely related to the research by Pelsser (2010), Pelsser \& Stadje (2014) and Pelsser \& Ghalehjooghi (2016). A detailed comparison is presented at the end of Section 5 .

In this paper we use the one-period valuation operator (3.1), but we face the problem of hedging annuity, death and survival benefits from (2.5). Let us discuss the hedging portfolio and the hedging problem for the benefit stream $B$. We note that the valuation operator (3.1) quantifies the non-hedgeable risks and measures the capital deficiency only at the terminal time $T$. We follow this approach. We separate the benefit stream $B$, which consists of benefits to be paid in $(0, T]$, into two parts. Let $B(0, T)$ denote the benefits from $B$ to be paid in $(0, T]$ excluding the terminal benefits to be paid at time $T$, and $\triangle B(T)$ denote the terminal benefits from $B$ to be paid at time $T$. We clearly have

$$
\begin{aligned}
B(0, T) & =\int_{0}^{T}(n-N(t-)) A(t, Y(t), F(t)) d t+\int_{0}^{T} D(t, Y(t), F(t)) d N(t), \\
\triangle B(T) & =(n-N(T)) S(Y(T), F(T)), \\
B(T) & =B(0, T]=B(0, T)+\triangle B(T) .
\end{aligned}
$$

Let $\theta=(\theta(t), 0 \leq t \leq T)$ denote an $\mathbb{F}$-predictable stochastic process which describes the amount invested in the risky asset $Y$. Let $V^{\theta}=\left(V^{\theta}(t), 0 \leq\right.$ $t \leq T$ ) denote the value of the hedging portfolio under the strategy $\theta$. The process $V^{\theta}$ can also be interpreted as the wealth process of the insurer. The dynamics of the hedging portfolio $V^{\theta}$ is described with the SDE:

$$
\begin{aligned}
d V^{\theta}(t)= & \theta(t)\left(\mu_{Y} d t+\sigma_{Y} d W_{Y}(t)\right)+\left(V^{\theta}(t)-\theta(t)\right) r d t \\
& -(n-N(t-)) A(t, Y(t), F(t)) d t-D(t, Y(t), F(t)) d N(t),
\end{aligned}
$$

and the terminal claims $\triangle B(T)$ are subtracted from $V^{\theta}(T)$ at time $T$. The hedging portfolio $V^{\theta}$ is continuously rebalanced with the dynamic hedging 
strategy $\theta$. The hedging strategy $\theta$ is continuously updated with the new information on the financial and insurance risks. The goal is to construct a hedging portfolio, given by (3.4), such that the hedging error at the terminal time $T$ for the terminal claims $\triangle B(T)$ is minimal in a mean-square sense.

In this paper we construct the optimal dynamic hedging strategy in the following way. Let $\mathbb{Q}$ denote an element of the set of equivalent martingale measures for the combined insurance and financial market, which is defined in (2.14). We find the hedging portfolio and the hedging strategy which minimize the mean-square hedging error at the terminal time under an equivalent martingale measure, i.e. we solve:

$$
\begin{aligned}
& \inf _{\theta} \mathbb{E}^{\mathbb{Q}}\left[\left|\triangle B(T)-V^{\theta}(T)\right|^{2}\right], \quad \mathbb{Q} \sim \mathbb{P}, \\
& V^{\theta} \text { satisfies the dynamics } 3.4 .
\end{aligned}
$$

The optimal hedging strategy and the optimal hedging portfolio are denoted by $\theta_{B(0, T), \triangle B(T)}^{*}$ and $V_{B(0, T), \triangle B(T)}^{*}$, or simply by $\theta_{B}^{*}$ and $V_{B}^{*}$. We will mostly use $B(0, T)$ and $\triangle B(T)$ instead of $B$ (or $B(0, T]$ ), since we need to separate the claims as in 3.3 .

Proposition 3.1. We consider the claims from the process $B$, which are separated in accordance with (3.3). Suppose that (A1)-(A4) hold and let us choose an equivalent martingale measure $\mathbb{Q}$ defined in (2.14). We assume there exist functions $\left(v^{k}\right)_{k=0, \ldots, n}$

$$
\begin{aligned}
v^{k}(t, y, f)= & \mathbb{E}_{t, y, f, k}^{\mathbb{Q}}\left[\int_{t}^{T} e^{-r(u-t)} d B(u)\right], \\
& (t, y, f) \in[0, T] \times(0, \infty) \times(0, \infty), k \in\{0, \ldots, n\},
\end{aligned}
$$

such that $v^{k} \in \mathcal{C}^{1,2,2}([0, T) \times(0, \infty) \times(0, \infty)) \cup \mathcal{C}([0, T] \times(0, \infty) \times(0, \infty))$, for each $k \in\{0, \ldots, n\}$.

We consider the optimization problem (3.5). The initial value of the hedging portfolio is given by

$$
V_{B(0, T), \triangle B(T)}^{*}(0)=v^{n}(0, Y(0), F(0))
$$


and the optimal dynamic hedging strategy is given by

$$
\begin{aligned}
\theta_{B(0, T), \triangle B(T)}^{*}(t)= & v_{y}^{J(t-)}(t, Y(t), F(t)) Y(t) \\
& +v_{f}^{J(t-)}(t, Y(t), F(t)) F(t) \frac{\sigma_{F}}{\sigma_{Y}} \rho, \quad 0 \leq t \leq T .
\end{aligned}
$$

Remark 3.1. a) The expected value $\mathbb{E}_{t, y, f, k}[\cdot]$ denotes the conditional expected value $\mathbb{E}[\cdot \mid Y(t)=y, F(t)=f, J(t)=k]$.

b) The collection of functions $\left(v^{k}\right)_{k=0, \ldots, n}$ gives us the arbitrage-free price of the benefit stream $B$ under the pre-specified equivalent martingale measure $\mathbb{Q}$. It is standard in financial mathematics to assume that such functions are smooth, or that the solutions to the PDEs used for option pricing are smooth, and satisfy some growth conditions, see e.g. Heath ES Schweizer (2000), Proposition 4.3 in El Karoui et al. (1997) or Proposition 1 in Cont E6 Voltchkova (2005).

c) Assumption (A4) is not needed in the proof, but this assumption is very reasonable, as already discussed, and we will need it in Proposition 3.4.

The quadratic hedging objective (3.5) under an equivalent martingale measure might be criticized since the insurer is interested in valuating losses and profits in the real-world. One can construct the hedging portfolio and the dynamic hedging strategy by minimizing the mean-square hedging error under the real-world measure:

$$
\begin{aligned}
& \inf _{\theta} \mathbb{E}^{\mathbb{P}}\left[\left|\triangle B(T)-V^{\theta}(T)\right|^{2}\right], \\
& V^{\theta} \text { satisfies the dynamics } 3.4,
\end{aligned}
$$

as in Dhaene et al. (2017), Barigou \& Dhaene (2018), Barigou et al. (2018).

Proposition 3.2. We consider the claims from the process $B$, which are separated in accordance with (3.3). Let (A1)-(A4) hold and let $\hat{\mathbb{Q}}$ denote the unique equivalent martingale measure for the tradeable risky asset $Y$ defined in 2.18). We assume there exist functions $\left(v^{k}\right)_{k=0, \ldots, n}$

$$
\begin{aligned}
v^{k}(t, y, f)= & \mathbb{E}_{t, y, f, k}^{\hat{\mathbb{Q}}}\left[\int_{t}^{T} e^{-r(u-t)} d B(u)\right], \\
& (t, y, f) \in[0, T] \times(0, \infty) \times(0, \infty), k \in\{0, \ldots, n\},
\end{aligned}
$$


such that $v^{k} \in \mathcal{C}^{1,2,2}([0, T) \times(0, \infty) \times(0, \infty)) \cup \mathcal{C}([0, T] \times(0, \infty) \times(0, \infty))$, for each $k \in\{0, \ldots, n\}$.

We consider the optimization problem (3.8). The initial value of the hedging portfolio is given by

$$
V_{B(0, T), \triangle B(T)}^{*}(0)=v^{n}(0, Y(0), F(0))
$$

and the optimal dynamic hedging strategy is given by

$$
\begin{gathered}
\theta_{B(0, T), \triangle B(T)}^{*}(t)=v_{y}^{J(t-)}(t, Y(t), F(t)) Y(t)+v_{f}^{J(t-)}(t, Y(t), F(t)) F(t) \frac{\sigma_{F}}{\sigma_{Y}} \rho \\
-\left(\frac{\mu_{Y}-r}{\sigma_{Y}^{2}}\right)\left(V_{B(0, T), \triangle B(T)}^{*}(t-)-v^{J(t-)}(t, Y(t), F(t))\right) \quad 0 \leq t \leq T .
\end{gathered}
$$

Let us compare the optimal hedging strategies (3.10) and (3.7).

Proposition 3.3. (i) The optimal dynamic hedging strategy from Proposition 3.1 derived by minimizing the mean-square hedging error under a martingale measure is market-consistent and actuarial,

(ii) The optimal dynamic hedging strategy from Proposition 3.2 derived by minimizing the mean-square hedging error under the real-world measure is market-consistent but not actuarial.

Remark 3.2. In the literature we can also find hedging strategies derived by minimizing a time-consistent mean-variance hedging error and hedging strategies derived by minimizing an instantaneous mean-variance hedging error (both under the real-world measure), see e.g. Hu et al. (2012) and Chapter 10.4 in Delong (2013). Such strategies are market-consistent, but they are not actuarial. The proof is beyond the scope of this paper.

The proposition and the remark above justify our choice of the hedging error under an equivalent martingale measure as the objective used in constructing the dynamic hedging strategy for the benefit stream $B$, at least in the class of quadratic hedging errors. To support our choice we also note that the optimal dynamic hedging strategy from Proposition 3.1 is a delta-hedging strategy - a type of hedging strategies commonly used in practice. Hence, in 
the sequel we assume that the hedging strategy and the hedging portfolio in the valuation operator (3.1) are derived by solving the optimization problem (3.5).

Proposition 3.4. Let us consider the one-period valuation operator (3.1) where the initial value of the hedging portfolio and the dynamic hedging strategy are characterized in Proposition 3.1. We assume that the one-period actuarial valuation operator $\pi$ satisfies the conditions of normalization and translation-invariance:

$$
\pi(0)=0, \quad \pi(\xi+a)=\pi(\xi)+a,
$$

for any random variable $\xi$ and constant a. The one-period valuation operator (3.1) is market-consistent and actuarial, hence it is fair.

We find it more convenient to represent the actuarial valuation operator $\pi$ as the expected value operator under the real-world measure $\mathbb{P}$ and the actuarial risk margin, i.e. we assume

$$
\pi(\xi)=\mathbb{E}^{\mathbb{P}}[\xi]+R M[\xi]
$$

where the risk margin valuation operator $R M$ is an actuarial risk margin valuation operator which satisfies $(2.9)$. The actuarial risk margin $R M$ fulfills the conditions implied by (3.11), i.e.

$$
R M[0]=0, \quad R M[\xi+a]=R M[\xi] .
$$

We focus on standard deviation and variance under $\mathbb{P}$ as the one-period actuarial risk margins.

\section{The multi-period iterated valuation opera- tor}

Let us consider the one-period valuation operator $\varrho$ from Proposition 3.4 . In this section we introduce the multi-period valuation operator by iterating 
the one-period valuation operator $\varrho$. Let $\mathcal{T}=\{0, h, \ldots, T-h, T\}$ with fixed $h$. Let us recall that by $\varphi_{B(t, T]}(t)$ we denote the price at time $t$ of the future claims from the process $B$. The price $\varphi_{B(t, T]}(t)$ at $t \in \mathcal{T}$ is defined by the following backward iterations:

$$
\begin{aligned}
\varphi_{\triangle B(T)}(T)= & \triangle B(T), \\
\varphi_{B(t, T]}(t)= & \varrho_{t}\left(\int_{t}^{t+h} d \tilde{B}(s)\right), \quad t=0, h, \ldots, T-h, \\
\tilde{B}(s)= & \int_{t}^{s}(n-N(u-)) A(u, Y(u), F(u)) d u \\
& +\int_{t}^{s} D(u, Y(u), F(u)) d N(u) \\
& +\varphi_{B(t+h, T]}(t+h) \mathbf{1}\{s=t+h\}, \quad t \leq s \leq t+h,
\end{aligned}
$$

where the valuation operator $\varrho_{t}$ is defined in Proposition 3.4 , and it is now defined conditional on the information available in the filtration $\mathcal{F}_{t}$. As in (3.3) we separate the benefit stream $\tilde{B}$ into $\tilde{B}(t, t+h)$ and $\triangle \tilde{B}(t+h)$. We have

$$
\begin{aligned}
\tilde{B}(t, t+h)= & \int_{t}^{t+h}(n-N(u-)) A(u, Y(u), F(u)) d u \\
& +\int_{t}^{t+h} D(u, Y(u), F(u)) d N(u)=B(t, t+h), \\
\triangle \tilde{B}(t+h)= & \varphi_{B(t+h, T]}(t+h) .
\end{aligned}
$$

In the sequel $\varphi_{B(t, T]}(t)$ is simply denoted by $\varphi(t)$.

In order to apply the iterations (4.1) and calculate the price $\varphi(t)$ at $t \in \mathcal{T}$ we have to solve a sequence of optimal hedging problems of the form 3.5 in our multi-period model. The insurer, in the period $[t, t+h]$, has to optimally hedge the claims from the process $B$ which arrive on $(t, t+h]$ and the value of the future claims $\varphi(t+h)$ at the terminal time $t+h$. The solutions to these hedging problems are given by Proposition 3.1. By the Markov property we 
have that $\varphi(t)=\varphi^{J(t)}(t, Y(t), F(t))$. Let

$$
\begin{aligned}
& v^{k}(s, y, f) \\
& =\mathbb{E}_{s, y, f, k}^{\mathbb{Q}}\left[\int_{s}^{t+h} e^{-r(u-s)} d B(u)+e^{-r(t+h-s)} \varphi^{J(t+h)}(t+h, Y(t+h), F(t+h))\right], \\
& \quad(s, y, f) \in[t, t+h] \times(0, \infty) \times(0, \infty), k \in\{0, \ldots, n\} .
\end{aligned}
$$

By Proposition 3.1, the optimal hedging strategy $\theta_{B(t, t+h), \varphi(t+h)}^{*}$ for the period $[t, t+h]$ is given by

$$
\begin{aligned}
\theta_{B(t, t+h), \varphi(t+h)}^{*}(s)= & v_{y}^{J(s-)}(s, Y(s), F(s)) Y(s) \\
& +v_{f}^{J(s-)}(s, Y(s), F(s)) F(s) \frac{\sigma_{F}}{\sigma_{Y}} \rho, \quad t \leq s \leq t+h .(4.3
\end{aligned}
$$

By (4.1) and Proposition 3.4 we study the following iterations

$$
\begin{aligned}
\varphi(t)= & V_{B(t, t+h), \varphi(t+h)}^{*}(t) \\
& +\pi_{t}\left(\left(\varphi(t+h)-V_{B(t, t+h), \varphi(t+h)}^{*}(t+h)\right) e^{-r h}\right), \quad t=0, h, \ldots, T-h,
\end{aligned}
$$

where $V_{B(t, t+h), \varphi(t+h)}^{*}$ denotes the optimal hedging portfolio given by (3.4) on $[t, t+h]$ with the optimal hedging strategy $\theta_{B(t, t+h), \varphi(t+h)}^{*}$. Using the decomposition of the one-period actuarial valuation operator (3.12), we end up with the backward, discrete-time equation:

$$
\begin{aligned}
\varphi(t) & =V_{B(t, t+h), \varphi(t+h)}^{*}(t)+\mathbb{E}^{\mathbb{P}}\left[\left(\varphi(t+h)-V_{B(t, t+h), \varphi(t+h)}^{*}(t+h)\right) e^{-r h} \mid \mathcal{F}_{t}\right] \\
& +R M\left[\left(\varphi(t+h)-V_{B(t, t+h), \varphi(t+h)}^{*}(t+h)\right) e^{-r h} \mid \mathcal{F}_{t}\right], \quad t=0, h, \ldots, T-h
\end{aligned}
$$

Let us introduce the discrete-time process

$$
X^{[t, t+h]}(s)=\varphi(s)-V_{B(t, t+h), \varphi(t+h)}^{*}(s), \quad s \in\{t, t+h\} .
$$

The process $\varphi$ should give us a fair price of the future benefits, or in other words it should give us the value of the technical provision the insurer should hold in order to cover the future benefits. Consequently, the process $N A V(s)=-X(s)$ determines the excess of the assets over the technical 
provision, and is called the net asset value. The net asset value $N A V$, derived from (4.5), results from valuating the future benefits with the operator $\varphi$, rebalancing the available wealth with the hedging strategy $\theta^{*}$ and covering the arriving benefits from $B$. Using the assumptions on the actuarial risk margin from Proposition 3.4, we can deduce the key pricing equation in our multi-period, discrete-time model with dynamic hedging in the sub-periods:

$$
\begin{aligned}
& \mathbb{E}\left[X^{[t, t+h]}(t+h) e^{-r h}-X^{[t, t+h]}(t) \mid \mathcal{F}_{t}\right] \\
& \quad+R M\left[X^{[t, t+h]}(t+h) e^{-r h}-X^{[t, t+h]}(t) \mid \mathcal{F}_{t}\right]=0, \quad t=0, h, \ldots, T-h,
\end{aligned}
$$

or

$$
\pi_{t}\left(X^{[t, t+h]}(t+h) e^{-r h}-X^{[t, t+h]}(t)\right)=0, \quad t=0, h, \ldots, T-h .
$$

In terms of net asset value, this can be written as

$$
\pi_{t}\left(N A V(t)-N A V(t+h) e^{-r h}\right)=0, \quad t=0, h, \ldots, T-h .
$$

From (4.7) we conclude that our iterated valuation principle (4.1) yields a price of the future benefits at times $t=0, h, \ldots, T-h$ such that the change in the net asset value in each sub-period $[t, t+h]$, after the application of the hedging strategy to the hedgeable part of the benefits, has an actuarial price equal to zero.

We remark that conditions 4.6)-(4.7) on the net asset value can also be expressed as

$$
\begin{aligned}
\mathbb{E} & {\left[N A V(t+h) e^{-r h} \mid \mathcal{F}_{t}\right]-N A V(t) } \\
& =R M\left[N A V(t)-N A V(t+h) e^{-r h} \mid \mathcal{F}_{t}\right] .
\end{aligned}
$$

From an asset-liability point of view, any profitable insurance company should satisfy

$$
\mathbb{E}\left[N A V(t+h) e^{-r h} \mid \mathcal{F}_{t}\right]-N A V(t)>0,
$$

which means that we expect that the insurance company grows at a rate higher than the risk-free rate $r$. Clearly, the growth rate is related to the premiums collected. From (4.8) we observe that the expected growth rate is 
related to the value of the risk margin which covers the non-hedgeable risks, since

$$
\begin{aligned}
& R M\left[N A V(t)-N A V(t+h) e^{-r h} \mid \mathcal{F}_{t}\right] \\
& \quad=R M\left[\left(\varphi(t+h)-V_{B(t, t+h), \varphi(t+h)}^{*}(t+h)\right) e^{-r h} \mid \mathcal{F}_{t}\right]
\end{aligned}
$$

The higher the risk margin (which is a part of the premium - the price $\varphi$ ), the higher the expected growth of the net asset value. Let us note that in practice the insurer at time $t+h$ should earn the risk margin which is included in the technical provision at time $t$, since the risk margin represents a safety buffer over the best estimate of the liability. Clearly, the higher the risk margin set at time $t$ as a part of the technical provision, the higher the expected surplus of the technical provision at time $t+h$ over the claims $(B(t, t+h), \varphi(t+h))$ paid in $(t, t+h]$, and the higher the expected profit earned by the insurance company (we assume that the technical provision is financed with the premiums collected from the policyholders). This property is reflected in our equations (4.8)-(4.10) and agrees with intuition.

In practical applications our valuation operator would work as follows. At time $t$ the insurer sets the hedging portfolio $V_{B(t, t+h), \varphi(t+h)}^{*}(t)$ on the asset side. The valuation operator (4.4) gives us the price of the future benefits or, in other words, the technical provision which the insurer should set at time $t$ on the liability side. Since $\varphi_{B(t, T]}(t)>V_{B(t, t+h), \varphi(t+h)}^{*}(t)$ due to the actuarial risk margin applied to the non-hedgeable risks (and possible differences between the expected value of the future benefits and the expected future value of the hedging portfolio), the insurer must set an additional capital on the asset side equal to $\varphi_{B(t, T]}(t)-V_{B(t, t+h), \varphi(t+h)}^{*}(t)$ at time $t$. This additional capital is invested in the risk-free bank account. The net asset value at time $t$ is equal to zero. During the period $(t, t+h]$ the hedging portfolio $V_{B(t, t+h), \varphi(t+h)}^{*}$ is rebalanced with the strategy $\theta_{B(t, t+h), \varphi(t+h)}^{*}$, the capital $\varphi_{B(t, T]}(t)-V_{B(t, t+h), \varphi(t+h)}^{*}(t)$ earns the risk-free rate and the insurer covers the benefits from the process $\int_{t}^{t+h} d B(s)$. At time $t+h$ the price of the future benefits $\varphi_{B(t+h, T]}(t+h)$ and the net asset value are recalculated. The capital 
from the risk margin, set at time $t$, will be used only in adverse scenarios to cover the non-hedgeable benefits from $\int_{t}^{t+h} d B(s)$ and the non-hedgeable change in the price of the future benefits: $\varphi_{B(t+h, T]}(t+h)-\varphi_{B(t, T]}(t)$. In the average scenario the insurer will earn the risk margin, accumulated with the risk-free rate, in the period $[t, t+h]$. The equations (4.7)-(4.10) confirm what we observe in practice. We remark that adding the additional capital $\varphi_{B(t, T]}(t)-V_{B(t, t+h), \varphi(t+h)}^{*}(t)$ to the assets and investing it in the risk-free bank account does not change $N A V(t+h) e^{-r h}-N A V(t)$, hence (4.8)-(4.10) remain valid.

Finally, let us recall that under Solvency II regulation, the solvency capital requirement over one year $(h=1)$ at time $t$ is defined by

$$
\mathbb{P}\left(N A V(t)-N A V(t+1) e^{-r} \geq S C R_{t} \mid \mathcal{F}_{t}\right)=\beta,
$$

with $\beta=0.5 \%$ as the confidence level. This can be written as

$$
\operatorname{VaR}_{1-\beta}\left[N A V(t)-N A V(t+1) e^{-r} \mid \mathcal{F}_{t}\right]=S C R_{t} .
$$

If the actuarial valuation $\pi_{t}$ in (4.1) is chosen to be the Value-at-Risk at confidence level $1-\beta$, from (4.7) we find that

$$
\begin{aligned}
0 & =\pi_{t}\left(N A V(t)-N A V(t+1) e^{-r}\right) \\
& =V_{a} R_{1-\beta}\left[N A V(t)-N A V(t+1) e^{-r} \mid \mathcal{F}_{t}\right],
\end{aligned}
$$

which means that $S C R_{t}$ is zero by 4.12 . This conclusion is obvious. If the insurer already prices the benefits with the actuarial risk margin such that the capital given by the operator (4.4) is sufficient to cover the benefits at the confidence level $\beta=0.5 \%$, then there is no need to keep additional solvency capital above the technical provision determined by (4.4). 


\section{Continuous-time limit of the multi-period iterated valuation operator and the continuous- time valuation operator}

In the previous section we define the price $\varphi_{B(t, T]}(t)$ of the future benefits from the process $B$ at times $t \in\{0, h, \ldots, T-h, T\}$ by using the one-period valuation operator (3.1) and the iterations (4.1). We would like to extend the definition of the price $\varphi_{B(t, T]}(t)$ to all times $t \in[0, T]$. The goal is to take $h \rightarrow 0$ in 4.4 and transform the backward, discrete-time equation 4.4 into a continuous-time equation (differential equation) with a terminal condition.

We study the discrete-time pricing equation (4.6) which involves the net asset value of the insurer. We fix the period $[t, t+h]$. We extend 4.5 and we introduce the continuous-time process

$$
X^{[t, t+h]}(s)=\varphi(s)-V_{B(t, t+h), \varphi(t+h)}^{*}(s), \quad t \leq s \leq t+h,
$$

where $\varphi$ denotes the continuous-time valuation operator which gives the price of the future benefit payments from the process $B$ at any time $t \in[0, T]$. We characterize the continuous-time valuation operator $\varphi$ as a function which satisfies the continuous-time limit of the discrete-time pricing equation 4.6 as $h \rightarrow 0$.

By the Markov property we have that $\varphi(t)=\varphi^{J(t)}(t, Y(t), F(t))$. We can write the dynamics for the process $X^{[t, t+h]}$ on $[t, t+h]$. Applying Itô's lemma, 
we find

$$
\begin{aligned}
d X^{[t, t+}+ & h]=\left\{\left(\varphi_{t}^{J(s-)}(s, Y(s), F(s))+\varphi_{y}^{J(s-)}(s, Y(s), F(s)) Y(s) \mu_{Y}\right.\right. \\
& +\varphi_{f}^{J(s-)}(s, Y(s), F(s)) F(s) \mu_{F} \\
& +\varphi_{y f}^{J(s-)}(s, Y(s), F(s)) Y(s) F(s) \sigma_{Y} \sigma_{F} \rho \\
+ & \frac{1}{2} \varphi_{y y}^{J(s-)}(s, Y(s), F(s)) Y^{2}(s) \sigma_{Y}^{2}+\frac{1}{2} \varphi_{f f}^{J(s-)}(s, Y(s), F(s)) F^{2}(s) \sigma_{F}^{2} \\
& -\theta_{B(t, t+h), \varphi(t+h)}^{*}(s)\left(\mu_{Y}-r\right)-V_{B(t, t+h), \varphi(t+h)}^{*}(s) r \\
+ & J(s-) A(s, Y(s), F(s))\} d s \\
+ & \left(\varphi_{y}^{J(s-)}(s, Y(s), F(s)) Y(s)-\theta_{B(t, t+h), \varphi(t+h)}^{*}(s)\right) \sigma_{Y} d W_{Y}(s) \\
+ & \varphi_{f}^{J(s-)}(s, Y(s), F(s)) F(s) \sigma_{F} d W_{F}(s)+D(s, Y(s), F(s)) d N(s) \\
+ & \left(\varphi^{J(s-)-1}(s, Y(s), F(s))-\varphi^{J(s-)}(s, Y(s), F(s))\right) d N(s) \\
= & X^{[t, t+h]}(s) r+\varphi_{t}^{J(s-)}(s, Y(s), F(s))+\varphi_{y}^{J(s-)}(s, Y(s), F(s)) Y(s) \mu_{Y} \\
+ & \varphi_{f}^{J(s-)}(s, Y(s), F(s)) F(s) \mu_{F} \\
+ & \varphi_{y f}^{J(s-)}(s, Y(s), F(s)) Y(s) F(s) \sigma_{Y} \sigma_{F} \rho \\
+ & \frac{1}{2} \varphi_{y y}^{J(s-)}(s, Y(s), F(s)) Y^{2}(s) \sigma_{Y}^{2}+\frac{1}{2} \varphi_{f f}^{J(s-)}(s, Y(s), F(s)) F^{2}(s) \sigma_{F}^{2} \\
- & \theta_{B(t, t+h), \varphi(t+h)}^{*}(s)\left(\mu_{Y}-r\right)-\varphi^{J(s-)}(s, Y(s), F(s)) r \\
+ & J(s-) A(s, Y(s), F(s)) \\
+ & \left(\varphi^{J(s-)-1}(s, Y(s), F(s))+D(s, Y(s), F(s))\right. \\
& \left.\left.-\varphi^{J(s-)}(s, Y(s), F(s))\right) J(s-) \lambda(s)\right\} d s \\
+ & \left(\varphi_{y}^{J(s-)}(s, Y(s), F(s)) Y(s)+\varphi_{f}^{J(s-)}(s, Y(s), F(s)) F(s) \frac{\sigma_{F}}{\sigma_{Y}} \rho\right. \\
& \left.-\theta_{B(t, t+h), \varphi(t+h)}^{*}(s)\right) \sigma_{Y} d W_{1}(s) \\
+ & \left(\varphi_{f}^{J(s-)}(s, Y(s), F(s)) F(s) \sigma_{F} \sqrt{1-)-1}(s, Y(s), F(s))+D(s, Y(s), F(s))\right. \\
& -\rho^{2} d W_{2}(s) \\
& \\
&
\end{aligned}
$$


Looking at (5.2), we can conclude that the risk of the process $X^{[t, t+h]}$ is induced by the three stochastic integrals with respect to the Brownian motions and the compensated counting process. From (4.3) we expect that

$$
\begin{aligned}
\theta_{B(t, t+h), \varphi(t+h)}^{*}(s) \sim & \varphi_{y}^{J(s-)}(s, Y(s), F(s)) Y(s) \\
& +\varphi_{f}^{J(s-)}(s, Y(s), F(s)) F(s) \frac{\sigma_{F}}{\sigma_{Y}} \rho, \quad t \leq s \leq t+h,
\end{aligned}
$$

for sufficiently small $h$. Consequently, for sufficiently small $h$, the risk of the process $X^{[t, t+h]}$ is only induced by the two stochastic integrals with respect to the Brownian motion $W_{2}$ and the compensated counting process $\tilde{N}$. These two stochastic integrals cannot be hedged by trading in $Y$ in the financial market - they model the non-hedgeable financial and insurance risks to which the insurer is exposed. Consequently, the actuarial risk margin valuation operator in the limit $h \rightarrow 0$ in (4.6) should only be applied to the second and third stochastic integral from (5.2).

It is common to measure the risk of a stochastic process with its quadratic variation, at least the risk of a process in a quadratic sense. Hence, we expect that the actuarial risk margin valuation operator in the limit $h \rightarrow 0$ in (4.6) should act on the integrals:

$$
\begin{aligned}
& {\left[\int_{t} \varphi_{f}^{J(u-)}(u, Y(u), F(u)) F(u) \sigma_{F} \sqrt{1-\rho^{2}} d W_{2}(u) d u\right]} \\
& =\int_{t}\left(\varphi_{f}^{J(u-)}(u, Y(u), F(u))\right)^{2} F^{2}(u) \sigma_{F}^{2}\left(1-\rho^{2}\right) d u,
\end{aligned}
$$

and

$$
\begin{aligned}
& {\left[\int _ { t } \left(\varphi^{J(u-)-1}(u, Y(u), F(u))+D(u, Y(u), F(u))\right.\right.} \\
& \left.\left.\quad-\varphi^{J(u-)}(u, Y(u), F(u))\right) d \tilde{N}(u)\right] \\
& =\int_{t}\left(\varphi^{J(u-)-1}(u, Y(u), F(u))+D(u, Y(u), F(u))\right. \\
& \left.\quad-\varphi^{J(u-)}(u, Y(u), F(u))\right)^{2} d N(u) .
\end{aligned}
$$

Moreover, the quadratic covariation between the last two stochastic integrals in (5.2) is zero. The first integral (5.3) measures (in a quadratic sense) the 
non-hedgeable risk that the value of the benefit payments changes due to a change in the independent component of the risky asset $F$. The integrand in (5.3) is the delta-hedging perfect replication strategy for the independent component of the risky asset $F$. This delta-hedging strategy cannot be applied by the insurer since $F$ is not traded. The second integral (5.4) measures (in a quadratic sense) the non-hedgeable risk that a policyholder dies: in case of death, the death benefit is paid and the price of the future claims is recalculated for the in-force policies. The integrand in (5.4) is the sum at risk to which the insurer is exposed in the event of the policyholder's death. The integrand in (5.4) can also be interpreted as the super-replication strategy for the insurance risk. This super-replication strategy cannot be applied by the insurer since its cost is too high to bear.

We can prove that the first term in 4.6 converges to

$$
\begin{aligned}
& \lim _{h \rightarrow 0} \frac{\mathbb{E}_{t, y, f, k}\left[X^{[t, t+h]}(t+h) e^{-r h}-X^{(t, t+h)}(t)\right]}{h} \\
&=\varphi_{t}^{k}(t, y, f)+\varphi_{y}^{k}(t, y, f) y r+\varphi_{f}^{k}(t, y, f) f\left(\mu_{F}-\frac{\mu_{Y}-r}{\sigma_{Y}} \sigma_{F} \rho\right) \\
&+\varphi_{y f}^{k}(t, y, f) y f \sigma_{Y} \sigma_{F} \rho+\frac{1}{2} \varphi_{y y}^{k}(t, y, f) y^{2} \sigma_{Y}^{2}+\frac{1}{2} \varphi_{f f}^{k}(t, y, f) f^{2} \sigma_{F}^{2} \\
&+k A(t, y, f) \\
&+\left(\varphi^{k-1}(t, y, f)+D(t, y, f)-\varphi^{k}(t, y, f)\right) k \lambda(t)-\varphi^{k}(t, y, f) r .
\end{aligned}
$$

If the variance is chosen as the one-period actuarial risk margin in (4.4), then one can prove that the second term in $(4.6)$ converges to

$$
\begin{aligned}
\lim _{h \rightarrow 0} & \frac{\operatorname{Var}_{t, y, f, k}\left[X^{[t, t+h]}(t+h) e^{-r h}-X^{[t, t+h]}(t)\right]}{h} \\
= & \left(\varphi_{f}^{k}(t, y, f)\right)^{2} f^{2} \sigma_{F}^{2}\left(1-\rho^{2}\right) \\
& +\left(\varphi^{k-1}(t, y, f)+D(t, y, f)-\varphi^{k}(t, y, f)\right)^{2} k \lambda(t) .
\end{aligned}
$$

If the standard deviation is chosen as the one-period actuarial risk margin, 
then we clearly have that

$$
\begin{aligned}
& \lim _{h \rightarrow 0} \sqrt{\frac{\operatorname{Var}_{t, y, f, k}\left[X^{[t, t+h]}(t+h) e^{-r h}-X^{[t, t+h]}(t)\right]}{h}} \\
& =\sqrt{\left(\varphi_{f}^{k}(t, y, f)\right)^{2} f^{2} \sigma_{F}^{2}\left(1-\rho^{2}\right)+\left(\varphi^{k-1}(t, y, f)+D(t, y, f)-\varphi^{k}(t, y, f)\right)^{2} k \lambda(t)} .
\end{aligned}
$$

As we expected, the continuous-time limits of the one-period variance and standard deviation risk margins in (4.6) depend on the processes which govern the quadratic variations (5.3)-(5.4).

We are now ready to state our key result.

Theorem 5.1. a) Let us consider the one-period valuation operator from Proposition 3.4 and the multi-period valuation operator defined by the backward iterations (4.1) of the one-period valuation operator with step $h$. We choose the equivalent martingale measure $\hat{\mathbb{Q}}$ from $(2.18)$ for the one-period mean-square hedging problem, and variance or standard deviation as the oneperiod actuarial risk margin:

$$
R M(\xi)=\frac{1}{2} \gamma \operatorname{Var}[\xi], \quad \text { or } \quad R M(\xi)=\frac{1}{2} \gamma \sqrt{h} \sqrt{\operatorname{Var}[\xi]},
$$

where $\gamma$ denotes a risk aversion coefficient. We investigate the discrete-time pricing equation (4.6).

b) Let us consider the PDEs

$$
\begin{aligned}
& \varphi_{t}^{k}(t, y, f)+\varphi_{y}^{k}(t, y, f) y r+\varphi_{f}^{k}(t, y, f) f\left(\mu_{F}-\frac{\mu_{Y}-r}{\sigma_{Y}} \sigma_{F} \rho\right) \\
& +\varphi_{y f}^{k}(t, y, f) y f \sigma_{Y} \sigma_{F} \rho+\frac{1}{2} \varphi_{y y}^{k}(t, y, f) y^{2} \sigma_{Y}^{2}+\frac{1}{2} \varphi_{f f}^{k}(t, y, f) f^{2} \sigma_{F}^{2} \\
& +k A(t, y, f)+\left(\varphi^{k-1}(t, y, f)+D(t, y, f)-\varphi^{k}(t, y, f)\right) k \lambda(t)-\varphi^{k}(t, y, f) r \\
& +\Phi^{k}\left(t, \varphi_{f}^{k}(t, y, f) f \sigma_{F} \sqrt{1-\rho^{2}}, \varphi^{k-1}(t, y, f)+D(t, y, f)-\varphi^{k}(t, y, f)\right)=0, \\
& \quad(t, y, f) \in[0, T) \times(0, \infty) \times(0, \infty), \\
& \varphi^{k}(T, y, f)=k S(y, f), \quad(y, f) \in(0, \infty) \times(0, \infty),
\end{aligned}
$$


for $k \in\{0, \ldots, n\}$, where $\Phi^{k}\left(t, x_{1}, x_{2}\right)=\frac{1}{2} \gamma\left(x_{1}^{2}+x_{2}^{2} k \lambda(t)\right)$ for the variance risk margin and $\Phi^{k}\left(t, x_{1}, x_{2}\right)=\frac{1}{2} \gamma \sqrt{x_{1}^{2}+x_{2}^{2} k \lambda(t)}$ for the standard deviation risk margin.

c) We assume that there exist unique solutions $\left(\varphi^{k}\right)_{k=0, \ldots, n}$ to the PDEs (5.8) such that $\varphi^{k} \in \mathcal{C}^{1,2,2}([0, T) \times(0, \infty) \times(0, \infty)) \cup \mathcal{C}([0, T] \times(0, \infty) \times(0, \infty))$ and the mixed derivatives $\varphi_{t x}^{k}, \varphi_{t f}^{k} \in \mathcal{C}([0, T) \times(0, \infty) \times(0, \infty))$, for each $k \in\{0, \ldots, n\}$. Moreover, we assume the growth conditions

$$
\begin{aligned}
\left|\varphi^{k}(t, y, f)\right| & \leq K\left(1+|y|^{p}+|f|^{p}\right), \quad \text { for } \quad \text { some } \quad p \geq 1 \\
\left|\varphi_{y}^{k}(t, y, f)\right|+\left|\varphi_{f}^{k}(t, y, f)\right| & \leq K\left(1+|y|^{p}+|f|^{p}\right), \quad \text { for } \quad \text { some } \quad p \geq 1
\end{aligned}
$$

Under a)-c), the continuous-time valuation operator $\varphi:=\left(\varphi^{k}\right)_{k=0, \ldots, n}$ determined by the PDEs (5.8) satisfies the continuous-time limit of the discretetime pricing equation (4.6) as $h \rightarrow 0$.

Remark 5.1. a) For the one-period valuation operator 3.1 we choose $\hat{\mathbb{Q}}$ in (3.5) when we solve the one-period mean-square hedging problems under the martingale measure. This choice of the martingale measure simplifies the proof. The proof of the theorem with an arbitrary equivalent martingale measure $\mathbb{Q}$ used in the one-period hedging problems is beyond the scope of this paper. More importantly, from a practical point of view we strongly believe that it is reasonable not to include any assumptions on the risk premiums for the non-hedgeable risks when we solve our hedging problems and set the value of the hedging portfolio as the price of the hedgeable part of the benefit stream (i.e. we should choose $\zeta(t)=\chi(t)=0$ in (2.14)). We recall that the choice of the risk premiums in (2.14) for pricing the non-hedgeable risks is subjective and we believe that these risk premiums should be implied by the subjective one-period actuarial valuation operator rather than being included in the equivalent martingale measure $\mathbb{Q}$ used for determining the hedging portfolio. This way we can disentangle hedgeable and non-hedgeable parts of the benefit stream and price them separately, see the interpretation of the one-period valuation operator (3.1). 
b) As pointed out by Pelsser \& Ghalehjooghi (2016), if we use the standard deviation risk margin, then we must use $\sqrt{h} \sqrt{\operatorname{Var}[\xi]}$ in order to have the convergence for $h \rightarrow 0$.

c) As far as the smoothness and growth conditions for $\varphi^{k}$ are concerned, we refer to the remark after Proposition 3.1. We point out that we refrain from making any growth assumptions on the second order derivatives as pointed out by Cont ES Voltchkova (2005).

We now discuss three crucial properties of the valuation operator $\varphi$ determined by the PDEs (5.8).

Theorem 5.2. Let us consider the continuous-time valuation operator $\varphi$ from Theorem 5.1.

(i) The valuation operator has the representation:

$$
\begin{aligned}
\varphi^{k}(t, y, f)=\mathbb{E}_{t, y, f, k}^{\hat{\mathbb{Q}}}\left[\int_{t}^{T} e^{-r(s-t)} d B(s)+\int_{t}^{T} e^{-r(s-t)} \Phi(s) d s\right], \\
(t, y, f) \in[0, T] \times(0, \infty) \times(0, \infty), k \in\{0, \ldots, n\}
\end{aligned}
$$

where $\Phi(s)$ is a shorthand notation for

$$
\begin{aligned}
\Phi^{J(s)} & \left(s, \varphi_{f}^{J(s)}(s, Y(s), F(s)) F(s) \sigma_{F} \sqrt{1-\rho^{2}},\right. \\
& \left.\varphi^{J(s)-1}(s, Y(s), F(s))+D(s, Y(s), F(s))-\varphi^{J(s)}(s, Y(s), F(s))\right),
\end{aligned}
$$

(ii) In addition we assume that the processes

$$
\begin{aligned}
\zeta_{V}(t)= & \frac{1}{2} \gamma \varphi_{f}^{J(t-)}(t, Y(t), F(t)) F(t) \sigma_{F} \sqrt{1-\rho^{2}}, \\
\chi_{V}(t)= & \frac{1}{2} \gamma\left(\varphi^{J(t-)-1}(t, Y(t), F(t))+D(t, Y(t), F(t))\right. \\
& \left.-\varphi^{J(t-)}(t, Y(t), F(t))\right),
\end{aligned}
$$

and

$$
\begin{aligned}
\zeta_{S D}(t) & =\frac{1}{2} \gamma \frac{\zeta_{V}(t)}{\sqrt{\left(\zeta_{V}(t)\right)^{2}+\left(\chi_{V}(t)\right)^{2} J(t-) \lambda(t)}}, \\
\chi_{S D}(t) & =\frac{1}{2} \gamma \frac{\chi_{V}(t)}{\sqrt{\left(\zeta_{V}(t)\right)^{2}+\left(\chi_{V}(t)\right)^{2} J(t-) \lambda(t)}}
\end{aligned}
$$


satisfy the conditions from 2.15). In this case, the valuation operator has the representation:

$$
\begin{aligned}
\varphi^{k}(t, y, f)= & \mathbb{E}_{t, y, f, k}^{\tilde{\mathbb{Q}}}\left[\int_{t}^{T} e^{-r(s-t)} d B(s)\right] \\
& (t, y, f) \in[0, T] \times(0, \infty) \times(0, \infty), k \in\{0, \ldots, n\},(5.1
\end{aligned}
$$

where $\tilde{\mathbb{Q}}$ denotes the equivalent martingale measure defined by (2.14) with the risk premiums $\left(\zeta_{V}, \chi_{V}\right)$ in the case of the one-period variance risk margin, and with the risk premiums $\left(\zeta_{S D}, \chi_{S D}\right)$ in the case of the one-period standard deviation risk margin. If $\chi_{V}(t)<0$ for some $t$, then the representation (5.10) holds only for sufficiently small $\gamma$ so that 2.15) is satisfied. The risk premiums satisfy the measurability assumption (A4).

(iii) The valuation operator is market-consistent and actuarial, hence it is fair.

Remark 5.2. We can show that the risk premiums $\left(\zeta_{S D}, \chi_{S D}\right)$ satisfy 2.15) under the assumptions from Theorem 5.1. The discussion when $\left(\zeta_{V}, \chi_{V}\right)$ satisfy 2.15) is beyond the scope of this paper. Point (ii) and the case $\chi_{V}(t)<0$ indicate that our continuous-time valuation operator may yield arbitrage prices, see Delong et al. (2018) for an example.

The representation $(5.9)$ is relevant for insurance, while the representation (5.10) is typical in finance. We focus on (5.9). If we look at the proof that leads to the PDEs (5.8), in particular, if we look at the limits (5.6)-(5.7), then we can conclude that the term $\Phi$ in the PDEs (5.8) is the continuous-time limit of the one-period actuarial risk margin $R M$. We can call $\Phi$ an instantaneous actuarial risk margin, and $\int \Phi(s)$ is the integrated instantaneous actuarial risk margin. The expected value of the integrated instantaneous actuarial risk margin is called the total actuarial risk margin. We note that the operator $\Phi$ becomes independent of the risky financial assets $(Y, F)$ when the valuation operator $\varphi$ is applied to an orthogonal process $B^{N}$, hence it satisfies (2.9), and this property justifies its name as an instantaneous actuarial risk margin, see also point (iii) in Theorem 5.2 . 
The representation (5.9) says that our continuous-time valuation operator $\varphi$ values liabilities as the best estimate of the liability plus the total actuarial risk margin for the liability:

$$
\begin{aligned}
\varphi_{B} & =\text { Fair Value of } B \\
& =\text { Best Estimate of } B+\text { Total Actuarial Risk Margin for } B .
\end{aligned}
$$

The best estimate of a liability is the expected value of the future claims from the liability, where the expected value is taken under the measure $\hat{\mathbb{Q}}$ given by 2.18). Let us recall that the equivalent martingale measure $\hat{\mathbb{Q}}$ results from choosing $\zeta(t)=\chi(t)=0$ in (2.14). It agrees with intuition that the best estimate assumptions for the non-tradeable financial and insurance risks should not include any risk premiums for these risks, see also Happ et al. (2015). We can observe that the best estimate of a liability contingent on the hedgeable financial risk coincides with the market cost of the investment portfolio which perfectly replicates the claims. The best estimate of a liability contingent on the independent, non-hedgeable financial and insurance risks is the expected cost, under the real-world measure $\mathbb{P}$, of the non-hedgeable claims to be paid. Intuitively, the best estimate of a liability contingent on the hedgeable and non-hedgeable financial and insurance risks consists of the market cost of the replicating portfolio for the hedgeable part of the claims and the expected, real-world, cost of the non-hedgeable claims left after the application of the hedging portfolio. The total actuarial risk margin, or the integrated instantaneous actuarial risk margin, gives us the capital which the insurer will need to set aside during the duration of the insurance portfolio in order to cover the non-hedgeable financial and insurance risks in adverse scenarios. In contrast, the one-period actuarial risk margin, from which we start in 4.1, provides the capital which the insurer needs to set aside for the next period of length $h$ in order to cover the non-hedgeable risks in adverse scenarios. These interpretations agree with valuation rules from the Solvency II Directive.

The calculations leading to Theorem 5.1 allow us to define the hedging 
strategy which underlies the continuous-time limit of the multi-period iterated valuation operator 4.1).

Theorem 5.3. Let us consider the continuous-time valuation operator $\varphi$ from Theorem 5.1. The hedging strategy which underlies the valuation $\varphi$ is given by

$\vartheta^{*}(t)=\varphi_{y}^{J(t-)}(t, Y(t), F(t)) Y(t)+\varphi_{f}^{J(t-)}(t, Y(t), F(t)) F(t) \frac{\sigma_{F}}{\sigma_{Y}} \rho, \quad 0 \leq t \leq T$.

The hedging strategy $\vartheta^{*}$ is market-consistent and actuarial, hence it is fair.

Equation (5.2) gives us the dynamics of the net asset value in the period $[t, t+h]$ if the optimal hedging strategy $\theta_{B(t, t+h), \varphi(t+h)}^{*}$ is applied on $[t, t+$ $h$. Let us derive the dynamics of the net asset value in the whole period $[0, T]$ during which the available assets are continuously rebalanced with the hedging strategy $\vartheta^{*}$ and the liabilities are continuously priced with the valuation operator $\varphi$. Let $X(s)=\varphi(s)-V^{*}(s)$, where $\varphi$ denotes the valuation operator defined in Theorem 5.1 and $V^{*}$ is the hedging portfolio given by (3.4) under the strategy $\vartheta^{*}$ from Theorem 5.3. From (5.2) and (5.8) we can deduce the dynamics of $X(s)$ :

$$
\begin{aligned}
d X(s)= & X(s) r d s-\Phi(s) d s \\
& +\varphi_{f}^{J(s-)}(s, Y(s), F(s)) F(s) \sigma_{F} \sqrt{1-\rho^{2}} d W_{2}(s) \\
+ & \left(\varphi^{J(s-)-1}(s, Y(s), F(s))+D(s, Y(s), F(s))\right. \\
& \left.\quad-\varphi^{J(s-)}(s, Y(s), F(s))\right) d \tilde{N}(s), \quad 0 \leq s \leq T .
\end{aligned}
$$

Let us recall that $N A V(t)=-X(t)$. By the martingale property of the stochastic integrals we find

$$
\begin{aligned}
\mathbb{E} & {\left[N A V(t) e^{-r(t-s)} \mid \mathcal{F}_{s}\right] } \\
& =N A V(s)+\mathbb{E}\left[\int_{s}^{t} e^{-r(u-s)} \Phi(u) d u \mid \mathcal{F}_{s}\right], \quad 0 \leq s \leq t \leq T .
\end{aligned}
$$

At each time $t \in[0, T)$, the insurer must hold an additional capital (the instantaneous actuarial risk margin) determined by $\Phi(t)$ which protects the 
insurer against adverse scenarios in the evolution of the non-hedgeable claims $d B(t)$ and the non-hedgeable change in the value of the claims $d \varphi(t)$ in an infinitesimal period of time $d t$. The evolution of the non-hedgeable risks is described with the two stochastic integrals in (5.11), see also (5.3)-(5.4). These two stochastic integrals describe the risk that the value of the benefit payments changes due to a change in the non-hedgeable, independent component of the risky asset $F$ and the risk that in the case of the nonhedgeable, independent event of the policyholder's death the insurer pays the death benefit and recalculates the value of the benefit payments for the in-force policies. Please note that the instantaneous actuarial risk margin $\Phi$ offsets the differentials of the stochastic integrals for the non-hedgeable risks in (5.11). At time $t=0$ the expected cost (i.e. the best estimate) of providing the additional capitals $\Phi$ till maturity of the insurance portfolio is equal to $\mathbb{E}^{\hat{\mathbb{Q}}}\left[\int_{0}^{T} e^{-r s} \Phi(s) d s\right]$ and is a part of the technical provision 5.9 - the total actuarial risk margin. As time $t$ goes by, the technical provision (the value of the benefit stream), the best estimate of the liability and the cost of financing the future instantaneous actuarial risk margins are recalculated. From (5.12) we see that the insurer earns, on average, a risk-free rate on the net asset value and the instantaneous actuarial risk margins accumulated with the risk-free rate. The instantaneous actuarial risk margins are released from the technical provision (5.9) as time passes and, on average, they are not used to cover the losses since the realized loss on the hedgeable risk is always zero and the expected loss on the non-hedgeable risks is also zero, both under $\mathbb{P}$ and $\hat{\mathbb{Q}}$ (the expected value of the stochastic integrals in (5.11) is zero). These interpretations in the continuous-time model agree with the interpretations we presented in Section 4 in the discrete-time, multi-period model.

Let us compare our results with the results from Pelsser (2010) and Pelsser $\&$ Ghalehjooghi (2016). These authors investigate the benefit stream

$$
B(t)=S(Y(T), F(T)) \mathbf{1}\{t=T\}
$$

which is a special case of our general benefit stream (2.5). Pelsser (2010) 
and Pelsser \& Ghalehjooghi (2016) propose a completely different one-period valuation operator $\varrho$, called a two-step valuation operator. They define the multi-period valuation operator by the iterations (4.1), take the limit $h \rightarrow 0$ and derive a PDE. Interestingly, for the benefit stream (5.13) and variance/standard deviation one-period valuation operator applied to the non-hedgeable risk our system of PDEs (5.8) reduces to a single PDE which agrees with the PDE derived by Pelsser (2010) and Pelsser \& Ghalehjooghi (2016). However, the valuation principle from these authors does not give a hedging strategy for the claims $B$, whereas our Theorem 5.3 does. Moreover, our results from Theorems 5.2,5.3 are linked to multi-period hedging problems from which the fair price of the claims $B$ is derived.

\section{Continuous-time valuation operator - be- yond quadratic actuarial risk margins}

When we look at the structure of the PDE (5.8), the discrete-time pricing equation (4.6) and the formulas (5.5)-(5.7), we can conclude that the last term $\Phi$ in the PDE is the continuous-time limit of the one-period actuarial risk margin $R M$, and the remaining terms are the continuous-time limit of the one-period expected value pricing principle. In the previous section we called $\Phi$ an instantaneous actuarial risk margin. The instantaneous actuarial risk margins $\Phi$ which we derived for the one-period standard deviation and variance risk margins act on $\varphi_{f}^{k}(t, y, f) f \sigma_{F} \sqrt{1-\rho^{2}}$ and $\varphi^{k-1}(t, y, f)+D(t, y, f)-\varphi^{k}(t, y, f)$. These two terms can be related to the

non-hedgeable part of the process $X^{[t, t+h]}$, or to the non-hedgeable part of the net asset value process, see (5.2)-(5.4). Since in the previous section we used quadratic actuarial risk margins to quantify the non-hedgeable risks, it is natural to use the quadratic variation of the process $X^{[t, t+h]}$ to define the instantaneous actuarial risk margin for the non-hedgeable risks. However, in the theory of stochastic processes and in applications, power variations are also used, see Theorem 2.2 in Barndorff-Nielsen et al. (2006) and Theorem 
$2.2 \mathrm{in} \operatorname{Jacod}(2008)$. Consequently, if we decide to quantify the non-hedgeable risks in $L^{q}$-norm, then we should use $q$-power variations of the process $X^{[t, t+h]}$. We would choose the instantaneous actuarial risk margin

$$
\Phi^{k}\left(t, x_{1}, x_{2}\right)=\frac{1}{2} \gamma\left(\left|x_{1}\right|^{q}+\left|x_{2}\right|^{q} k \lambda(t)\right)^{1 / q},
$$

and apply it to $\varphi_{f}^{k}(t, y, f) f \sigma_{F} \sqrt{1-\rho^{2}}, \varphi^{k-1}(t, y, f)+D(t, y, f)-\varphi^{k}(t, y, f)$.

Let us remark that Rosazza Gianin (2006) and Barrieu \& Karoui (2005), among others, develop dynamic risk measures which are defined as solutions to backward stochastic differential equations (BSDEs), see also Pelsser \& Stadje (2014). In their framework, our $\mathbb{F}$-adapted benefit stream $B$ would be valued at any time $t \in[0, T]$ with the process $\mathcal{Y}$ which is a solution to the BSDE

$$
\begin{aligned}
\mathcal{Y}(t) & =\int_{t}^{T} d B(s)+\int_{t}^{T}\left(g\left(s, \mathcal{Z}_{2}(s), \mathcal{U}(s)\right)-\frac{\mu_{Y}-r}{\sigma_{Y}} \mathcal{Z}_{1}(s)-\mathcal{Y}(s-) r\right) d s \\
& -\int_{t}^{T} \mathcal{Z}_{1} d W_{1}(s)-\int_{t}^{T} \mathcal{Z}_{2}(t) d W_{2}(s)-\int_{t}^{T} \mathcal{U}(t) d \tilde{N}(s), \quad 0 \leq t \leq T,
\end{aligned}
$$

where $g$ is interpreted as an instantaneous risk measure or a local preferencebased pricing rule for the non-hedgeable risks. Consequently, new dynamic risk measures can be constructed with BSDEs and instantaneous risk measures. From the proof of Proposition 3.1, we find that in our Markovian model the solution to the BSDE (6.1) is given by

$$
\begin{aligned}
\mathcal{Y}(t) & =\phi^{J(t)}(t, Y(t), F(t)) \\
& =\mathbb{E}^{\hat{\mathbb{Q}}}\left[\int_{t}^{T} e^{-r(s-t)} d B(s)+\int_{t}^{T} e^{-r(s-t)} g\left(s, \mathcal{Z}_{2}(s), \mathcal{U}(s)\right) d s \mid \mathcal{F}_{t}\right], \\
\mathcal{Z}_{1}(t) & =\phi_{y}^{J(t-)}(t, Y(t), F(t)) Y(t) \sigma_{Y}+\phi_{f}^{J(t-)}(t, Y(t), F(t)) F(t) \sigma_{F} \rho, \\
\mathcal{Z}_{2}(t) & =\phi_{f}^{J(t-)}(t, Y(t), F(t)) F(t) \sigma_{F} \sqrt{1-\rho^{2}}, \\
\mathcal{U}(t) & =\phi^{J(t-)-1}(t, Y(t), F(t))+D(t, Y(t), F(t))-\phi^{J(t-)}(t, Y(t), F(t)),
\end{aligned}
$$

and the dynamic risk measure $\phi$ induced by the BSDE has exactly the same representation as our continuous-time valuation operator (see point (i) of Theorem 5.2. . 
Consequently, we propose to price the benefit stream $B$ with the operator $\varphi$ which satisfies the system of PDEs:

$$
\begin{aligned}
& \varphi_{t}^{k}(t, y, f)+\varphi_{y}^{k}(t, y, f) y r+\varphi_{f}^{k}(t, y, f) f\left(\mu_{F}-\frac{\mu_{Y}-r}{\sigma_{Y}} \sigma_{F} \rho\right) \\
& +\varphi_{y f}^{k}(t, y, f) y f \sigma_{Y} \sigma_{F} \rho+\frac{1}{2} \varphi_{y y}^{k}(t, y, f) y^{2} \sigma_{Y}^{2}+\frac{1}{2} \varphi_{f f}^{k}(t, y, f) f^{2} \sigma_{F}^{2} \\
& +k A(t, y, f)+\left(\varphi^{k-1}(t, y, f)+D(t, y, f)-\varphi^{k}(t, y, f)\right) k \lambda(t)-\varphi^{k}(t, y, f) r \\
& +\Phi^{k}\left(\varphi_{f}^{k}(t, y, f) f \sigma_{F} \sqrt{1-\rho^{2}}, \varphi^{k-1}(t, y, f)+D(t, y, f)-\varphi^{k}(t, y, f)\right)=0 \\
& (t, y, f) \in[0, T) \times(0, \infty) \times(0, \infty), \\
& \varphi^{k}(T, y, f)=k S(y, f), \quad(y, f) \in(0, \infty) \times(0, \infty),
\end{aligned}
$$

for $k \in\{0, \ldots, n\}$, where $\Phi$ denotes some instantaneous actuarial risk margin applied to the non-hedgeable part of the insurer's net asset value process. The operator $\Phi$ locally quantifies the non-hedgeable risks which are present in the evolution of the insurer's net asset value. The instantaneous risk margin $\Phi$ should be interpreted as the continuous-time limit of the one-period risk margin $R M$ used in the multi-period iterated valuation operator (4.4). For continuous-time limits of some static risk measures we refer to Stadje (2010). Our previous calculations and elaborations justify the PDE (6.2). We can conclude that the valuation operator arising as the continuous-time limit of the multi-period iterated valuation operator (4.4) with the optimal meansquare hedging portfolio and an arbitrary one-period actuarial risk margin should satisfy the PDEs 6.2 with some function $\Phi$.

Proposition 6.1. Let us define the continuous-time valuation operator $\varphi$ with the PDEs 6.2 with an arbitrary function $\Phi:\{0, \ldots, n\} \times[0, T] \times \mathbb{R} \times \mathbb{R} \mapsto$ $[0, \infty)$ such that $\Phi^{k}(t, 0,0)=0$ and $\Phi$ satisfies the polynomial growth

$$
\left|\Phi^{k}\left(t, x_{1}, x_{2}\right)\right| \leq K\left(1+\left|x_{1}\right|^{p}+\left|x_{2}\right|^{p}\right), \quad \text { for some } p \geq 1 \text {. }
$$

In case all assumptions from Theorem 5.1 are satisfied, we have that the valuation operator $\varphi$ and the corresponding hedging strategy $\vartheta^{*}$ satisfy all properties from Theorems 5.2 and 5.3. 
Finally, we note that the standard deviation or variance instantaneous risk margin (quadratic variation) penalizes both losses and gains on the net asset value process (5.2). In applications, we might prefer to use asymmetric instantaneous actuarial risk margins $\Phi$ which only penalize losses on the net asset value process.

\section{Proofs of the results}

Proof of Proposition 3.1: Since the claims $A, D, S$ satisfy (A3) and $(Y, F)$ are geometric Brownian motions, we can show that $\mathbb{E}\left[|B(T)|^{q}\right]<\infty$ for all $q \geq 2$. Let $\mathcal{M}^{\zeta}, \chi$ denote the martingale (2.14) which defines $\mathbb{Q}$. We know that $\mathbb{E}\left[\left|\mathcal{M}^{\zeta, \chi}(T)\right|^{l}\right]<\infty$ for some $l>1$, and we can prove that $\mathbb{E}^{\mathbb{Q}}\left[|B(T)|^{q}\right]<\infty$ for all $q \geq 2$. The property of predictable representation gives us

$$
\begin{aligned}
\int_{0}^{T} e^{-r t} d B(t)= & \mathbb{E}^{\mathbb{Q}}\left[\int_{0}^{T} e^{-r t} d B(t)\right]+\int_{0}^{T} Z_{1}(t) d W_{1}^{\mathbb{Q}}(t) \\
& +\int_{0}^{T} Z_{2}(t) d W_{2}^{\mathbb{Q}}(t)+\int_{0}^{T} U(t) d \tilde{N}^{\mathbb{Q}}(t) .
\end{aligned}
$$

From Theorem 5.1 in El Karoui et al. (1997) we conclude that

$$
\mathbb{E}^{\mathbb{Q}}\left[\left(\int_{0}^{T}\left|Z_{1}(t)\right|^{2} d t\right)^{q / 2}\right]<\infty,
$$

for all $q \geq 2$. Since the martingale $\mathcal{N}^{\zeta^{\prime}}, \chi^{\prime}$ which defines the derivative $\frac{d \mathbb{P}}{d \mathbb{Q}} \mid \mathcal{F}_{t}=$ $\mathcal{N}^{\zeta^{\prime}, \chi^{\prime}}(t)$ also satisfies $\mathbb{E}\left[\left|\mathcal{N}^{\zeta^{\prime}}, \chi^{\prime}(T)\right|^{l}\right]<\infty$ for some $l>1$, we can show that the process $Z_{1}$ is square integrable under $\mathbb{P}$.

By Theorem 10.1.1 in Delong (2013) the optimal hedging strategy is given by $\theta_{B(0, T), \triangle B(T)}^{*}(t)=\frac{Z_{1}(t)}{\sigma_{Y}} e^{r t}$. Recalling the arguments from above and the function $v$ defined in this proposition, we deduce that the process $M(t)=$ $v^{J(t)}(t, Y(t), F(t)) e^{-r t}+\int_{0}^{t} e^{-r u} d B(u)$ is a $\mathbb{Q}$-martingale with the representation (7.1). Since $v$ is a smooth function on $[0, T-\epsilon] \times(0, \infty) \times(0, \infty)$, we can apply Itô's formula to the process $M$ on $[0, T-\epsilon]$. The form of the optimal hedging strategy $(3.7)$ on $[0, T-\epsilon]$ can be found by comparing the stochastic 
integral with respect to $W_{1}$ from $M$ with the stochastic integral with respect to $W_{1}$ from 7.1 . We have

$$
\begin{aligned}
& \mathbb{E}\left[\int_{0}^{T-\epsilon}\left|v_{y}^{J(t-)}(t, Y(t), F(t)) Y(t)+v_{f}^{J(t-)}(t, Y(t), F(t)) F(t) \frac{\sigma_{F}}{\sigma_{Y}} \rho\right|^{2} d t\right] \\
& \leq \mathbb{E}\left[\int_{0}^{T}\left|\frac{Z_{1}(t)}{\sigma_{Y}} e^{r t}\right|^{2} d t\right]<\infty,
\end{aligned}
$$

and we can define the strategy (3.7) on $[0, T]$ by the Fatou's lemma and $\epsilon \rightarrow 0$. We also conclude that the optimal hedging strategy is square integrable.

Proof of Proposition 3.2: We refer the reader to Chapter 10.2 in Delong (2013) and the proof of Proposition 3.1.

Proof of Proposition 3.3: (i) Let us consider $\tilde{B}(t)=B^{Y}(t)+B(t)$ where $B^{Y}$ is a hedgeable process and $B$ is an arbitrary process. By the additivity property of the expected value operator, we get $v_{\tilde{B}}^{k}(t, y, f)=v_{B^{Y}}^{k}(t, y, f)+$ $v_{B}^{k}(t, y, f)$, where $v$ is defined in Proposition 3.1. Moreover, $v_{B^{Y}}^{k}(t, y, f)=$ $v_{B^{Y}}(t, y)$. By the definition of the optimal hedging strategy $\theta_{\tilde{B}}^{*}$ from $(3.7)$, we conclude that condition 2.12 is satisfied. For an orthogonal process $B^{N}$ we have $v_{B^{N}}^{k}(t, y, f)=v_{B^{N}}^{k}(t)$ (since $\chi$ is $\mathbb{F}^{N}$-predictable and Markov by (A4)). Consequently, $\theta_{B^{N}}^{*}(t)=0$ and condition 2.13 is satisfied.

(ii) We use the results from the proof of (i). We note that the optimal hedging strategy from 3.10 is a linear function of the hedging portfolio, with a constant slope and an intercept which is additive with respect to the benefit stream $\tilde{B}=B^{Y}+B$. From the linear SDE (3.4) describing the dynamics of the hedging portfolio with the additive benefit stream $\tilde{B}=$ $B^{Y}+B$, we can conclude that $V_{\tilde{B}}^{*}(t)=V_{B^{Y}}^{*}(t)+V_{B}^{*}(t)$. By (3.10) we find $\theta_{\tilde{B}}^{*}(t)=\theta_{B^{Y}}^{*}(t)+\theta_{B}^{*}(t)$. Since $B^{Y}$ is hedgeable, $V_{B^{Y}}^{*}(T)=v_{B^{Y}}(T, Y(T))$ and 2.12 is satisfied.

We give an example showing that the optimal hedging strategy 3.10 is not actuarial. Let us assume that the insurer is exposed to the orthogonal process $B^{N}$ which only includes the constant survival benefits $S=1$. From Proposition 3.2 we can conclude that $v_{B^{N}}^{k}(t, y, f)=v_{B^{N}}^{k}(t), \theta_{B^{N}}^{*}(0)=0$ 
and $V_{B^{N}}^{*}(0)=\mathbb{E}^{\hat{\mathbb{Q}}}\left[e^{-r T}(n-N(T))\right]=n e^{-r T} e^{-\int_{0}^{T} \lambda(s) d s}$. However, at some latter time $t$ we may have $V_{B^{N}}^{*}(t)<\mathbb{E}^{\hat{\mathbb{Q}}}\left[e^{-r(T-t)}(n-N(T)) \mid \mathcal{F}_{t}\right]=(n-$ $N(t)) e^{-r(T-t)} e^{\left.-\int_{t}^{T} \lambda(s) d s\right)}=v_{B^{N}}^{J(t)}(t)$, since the insurance risk may evolve differently from the way the insurer expected at time $t=0$ and the riskfree investment is not sufficient to cover the reserve at time $t$ (less policyholders have died on $[0, t]$ than the insurer expected at time $t=0$ and $\left.N(t)<n\left(1-e^{-\int_{0}^{t} \lambda(s) d s}\right)\right)$. If $\mu_{Y}>r$, then the insurer can reduce the deficiency in the reserve and can reduce the mean-square hedging error at the terminal time $T$ by investing in the risky asset $Y$. Consequently, the optimal dynamic hedging strategy $(3.10)$ is not actuarial.

Proof of Proposition 3.4: Let us consider $\tilde{B}(t)=B^{Y}(t)+B(t)$ where $B^{Y}$ is a hedgeable process and $B$ is an arbitrary process. By Propositions 3.1 and 3.3 , we know that $\theta_{\tilde{B}}^{*}(t)=\theta_{B^{Y}}^{*}(t)+\theta_{B}^{*}(t)$ and the optimal hedging strategies do not depend on the value of the hedging portfolio. Consequently, from the linear SDE (3.4) describing the dynamics of the hedging portfolio with the additive benefit stream $\tilde{B}=B^{Y}+B$, we can conclude that $V_{\tilde{B}}^{*}(t)=$ $V_{B^{Y}}^{*}(t)+V_{B}^{*}(t)$. Since $B^{Y}$ can be perfectly replicated with $V_{B^{Y}}^{*}$, we have $\triangle \tilde{B}(T)-V_{\tilde{B}}^{*}(T)=\triangle B(T)-V_{B}^{*}(T)$. From 3.1 we deduce

$\varrho(\tilde{B})=V_{B^{Y}}^{*}(0)+V_{B}^{*}(0)+\pi\left(\left(\triangle B(T)-V_{B}^{*}(T)\right) e^{-r T}\right)=\rho(B)+V_{B^{Y}}^{*}(0)$.

By Proposition 3.1 we find $V_{B^{Y}}^{*}(0)=\mathbb{E}^{\mathbb{Q}}\left[\int_{0}^{T} e^{-r s} d B^{Y}(s)\right]=\mathbb{E}^{\hat{\mathbb{Q}}}\left[\int_{0}^{T} e^{-r s} d B^{Y}(s)\right]$. If $B(t)=0$, then $\varrho(B)=0$ by the first condition in 3.11 , and $\varrho(\tilde{B})=$ $\varrho\left(B^{Y}\right)=V_{B^{Y}}^{*}(0)$. We can conclude that the valuation operator 3.1 satisfies (2.8).

Let $B^{N}$ denote an orthogonal process. By Propositions 3.1 and $3.3 . \theta_{B^{N}}^{*}(t)=$ 0 and $V_{B^{N}}^{*}(T)=V_{B^{N}}^{*}(0) e^{r T}-\int_{0}^{T} e^{r(T-s)} d B^{N}(s)+\triangle B^{N}(T)$. Substituting the formula for $V_{B^{N}}^{*}(T)$ into the definition of the valuation operator (3.1), using the second condition in (3.11) and the assumption that $\pi$ is actuarial, we can easily prove that the valuation operator (3.1) satisfies (2.9).

Proof of Theorem 5.1: We study the terms on the left hand side of equa- 
tion (4.6) and we choose the variance as the one-period actuarial risk margin $R M$. We consider the process $X^{[t, t+h]}$ given by (5.1) where $\varphi$ denotes the continuous-time valuation operator. Let us fix $(t, y, f, k) \in[0, T) \times(0, \infty) \times$ $(0, \infty) \times\{0, \ldots, k\}$. We fix sufficiently small $h<1$ such that $t+h<T$ and we consider the interval $[t, t+h]$. Consequently, we always have $s \in[t, t+h]$. - Step 1: We collect some results which we will often use in the sequel. By equations (2.16)-(2.17), we have the following properties under the measures $\mathbb{P}$ and $\hat{\mathbb{Q}}$ : the processes $Y$ and $F$ are geometric Brownian motions, the counting process $N$ has intensity $\lambda(t)$ and $N$ is independent of $(Y, F)$. We consider $Y$, but the same results hold for $F$. Let $Y^{t, y}$ denote the process $Y$ which starts at time $t$ from $y$. We have $Y^{t, y}(s)=y e^{\mu(t-s)-\frac{1}{2} \sigma^{2}(s-t)+\sigma W(s-t)}=$ $y e^{\mu(t-s)} M^{t, 1}(s)$, and $M$ is an exponential martingale. By Doob's inequality we find that

$$
\begin{aligned}
\mathbb{E}\left[\sup _{s \in[t, t+h]}\left|Y^{t, y}(s)\right|^{q}\right] & \leq K|y|^{q} \mathbb{E}\left[\left|M^{t, 1}(t+h)\right|^{q}\right] \\
& =K|y|^{q} e^{\frac{1}{2}\left(q^{2}-q\right) \sigma^{2} h} \leq K|y|^{q}, \quad q \geq 2 .
\end{aligned}
$$

We can also show that

$$
\begin{aligned}
& \mathbb{E}\left[\sup _{s \in[t, t+h]}\left|Y^{t, y+\alpha}(s)-Y^{t, y}(s)\right|^{q}\right] \leq K|\alpha|^{q}, \quad q \geq 2, \\
& \mathbb{E}\left[\sup _{s \in[t, t+h]}\left|Y^{t, y}(s)-y\right|^{q}\right] \\
& =|y|^{q} \mathbb{E}\left[\sup _{s \in[t, t+h]}\left|e^{\mu(s-t)}\left(1+\int_{t}^{s} M^{t, 1}(u) \sigma d W(u)\right)-1\right|^{q}\right] \\
& \leq K|y|^{q} \mathbb{E}\left[\sup _{s \in[t, t+h]}\left\{\left|e^{\mu(s-t)}-1\right|^{q}+\left|e^{\mu(s-t)} \int_{t}^{s} M^{t, 1}(u) \sigma d W(u)\right|^{q}\right\}\right] \\
& \leq K|y|^{q}\left(h+\mathbb{E}\left[\left.\left.\left|\int_{t}^{t+h}\right| M^{t, 1}(u) \sigma\right|^{2} d u\right|^{q / 2}\right]\right) \\
& \leq K|y|^{q} h\left(1+\mathbb{E}\left[\sup _{u \in[t, t+h]}\left|M^{t, 1}(u)\right|^{q}\right]\right) \leq K|y|^{q} h, \quad q \geq 2,
\end{aligned}
$$

where we use the Burkholder-Davis-Gundy inequality. The same moment estimates hold under $\hat{\mathbb{Q}}$. We can note that the mapping $(t, y, s) \mapsto Y^{t, y}(s)$ is 
a.s continuous. Moreover, $\frac{d}{d y} Y^{t, y}(s)=\frac{Y^{t, y}(s)}{y}$. We can also prove that

$$
\begin{aligned}
\mathbb{E}\left[\sup _{s \in[t, t+h]}\left|J^{t, k}(s)-k\right|^{q}\right] & =\mathbb{E}\left[\left|J^{t, k}(t+h)-k\right|^{q}\right] \leq K \mathbb{E}\left[\left|J^{t, k}(t+h)-k\right|\right] \\
& =K \mathbb{E}\left[\int_{t}^{t+h} J^{t, k}(u) \lambda(u) d u\right] \leq K h, \quad q \geq 2 .
\end{aligned}
$$

- Step 2: We investigate the function 4.2. We define

$$
\begin{aligned}
& v^{k}(s, y, f) \\
& =\mathbb{E}_{s, y, f, k}^{\hat{\mathbb{Q}}}\left[\int_{s}^{t+h} e^{-r(u-s)} d B(u)+e^{-r(t+h-s)} \varphi^{J(t+h)}(t+h, Y(t+h), F(t+h))\right] \\
& =\mathbb{E}^{\hat{\mathbb{Q}}}\left[k \int_{s}^{t+h} e^{-r(u-s)} A\left(u, Y^{s, y}(u), F^{s, f}(u)\right) e^{-\int_{s}^{u} \lambda(z) d z} d u\right. \\
& \quad+k \int_{s}^{t+h} e^{-r(u-s)} D\left(u, Y^{s, y}(u), F^{s, y}(u)\right) e^{-\int_{s}^{u} \lambda(z) d z} \lambda(u) d u \\
& \left.\quad+e^{-r(t+h-s)} \sum_{i=0}^{k} \varphi^{i}\left(t+h, Y^{s, y}(t+h), F^{s, f}(t+h)\right) \operatorname{Pr}(J(t+h)=i \mid J(t)=k)\right],
\end{aligned}
$$

where we use the independence between $N$ and $(Y, F)$ from Step 1. The function $(s, y, f) \mapsto v^{k}(s, y, f)$ is finite and continuous on $[t, t+h] \times(0, \infty) \times(0, \infty)$ by Step 1), the growth and continuity conditions for $A, D, \varphi^{i}$, boundedness of $\lambda$ and the uniform integrability of $\left(\Lambda^{\tilde{s}, \tilde{y}, \tilde{f}}\right)_{(\tilde{s}, \tilde{y}, \tilde{f}) \in[s-\epsilon, s+\epsilon] \times[y-\epsilon, y+\epsilon] \times[f-\epsilon, f+\epsilon]}$ where $v^{k}(s, y, f)=\mathbb{E}^{\hat{\mathbb{Q}}}\left[\Lambda^{s, y, f}\right]$, see Lemma 2 from Heath \& Schweizer $(2000)$. From Theorem 1 in Heath \& Schweizer (2000) we can conclude that $v^{k} \in$ $\mathcal{C}^{1,2,2}([t, t+h) \times(0, \infty) \times(0, \infty)) \cup \mathcal{C}([t, t+h] \times(0, \infty) \times(0, \infty))$, for each $k \in\{0, \ldots, n\}$. Consequently, we can apply Proposition 3.1.

- Step 3: The hedging strategy $\theta_{B(t, t+h), \varphi(t+h)}^{*}$ is given by 4.3. Let us denote $\theta_{B(t, t+h), \varphi(t+h)}^{*}$ by $\theta^{*}$. We derive a more explicit formula for $\theta^{*}$. We will calculate the derivative $v_{y}^{k}$. The derivative $v_{f}^{k}$ can be calculated in the same 
way. We consider $\alpha<1$. Let

$$
\begin{aligned}
\nabla & A_{\alpha}(u) \\
& =\frac{A\left(u, Y^{s, y+\alpha}(u), F(u)\right)-A\left(u, Y^{s, y}(u), F(u)\right)}{\alpha}, \\
\nabla & \varphi_{\alpha}^{i} \\
& =\frac{\varphi^{i}\left(t+h, Y^{s, y+\alpha}(t+h), F(t+h)\right)-\varphi^{i}\left(t+h, Y^{s, y}(t+h), F(t+h)\right) \mid}{\alpha},
\end{aligned}
$$

for $u \in[s, t+h]$ and $i \in\{0, \ldots, n\}$. By the Lipshitz property of $A$ we have the estimate

$$
\left|\nabla A_{\alpha}(u)\right| \leq K \frac{\sup _{u \in[s, t+h]}\left|Y^{s, y+\alpha}(u)-Y^{s, y}(u)\right|}{\alpha} .
$$

By Fubini's theorem, we have that $\mathbb{E}^{\hat{\mathbb{Q}}}\left[\int_{s}^{t+h} \nabla A_{\alpha}(u) d u\right]=\int_{s}^{t+h} \mathbb{E}^{\hat{\mathbb{Q}}}\left[\nabla A_{\alpha}(u)\right] d u$. We can show that $\sup _{u \in[s, t+h]} \mathbb{E}^{\hat{\mathbb{Q}}}\left[\nabla A_{\alpha}(u)\right] \leq \sup _{u \in[s, t+h]} \sqrt{\mathbb{E} \hat{\mathbb{Q}}\left[\left|\nabla A_{\alpha}(u)\right|^{2}\right]} \leq$ $K$ by the moment estimates from Step 1 . Hence, by the dominated convergence theorem we can take the limit $\alpha \rightarrow 0$ under the integral $\int_{s}^{t+h} \mathbb{E}^{\hat{\mathbb{Q}}}\left[\nabla A_{\alpha}(u)\right] d u$. Using again the moment estimates from Step 1, we can deduce that

$$
\sup _{\alpha \in[0,1)} \mathbb{E}^{\hat{\mathbb{Q}}}\left[\left|\nabla A_{\alpha}(u)\right|^{2}\right] \leq K
$$

for each fixed $u \in[s, t+h]$. Consequently, by the uniform integrability of $\left(\nabla A_{\alpha}(u)\right)_{\alpha}$ we can take the limit $\alpha \rightarrow 0$ under $\mathbb{E}^{\hat{\mathbb{Q}}}\left[\nabla A_{\alpha}(u)\right]$ for each fixed $u \in$ $[s, t+h]$. Recall that $z \mapsto A(., z,$.$) is Lipschitz and has a countable number of$ non-differentiable points, the law of $Y^{t, y}(u)$ is absolutely continuous and $y \mapsto$ $Y^{t, y}(u)$ is differentiable by Step 1 . Therefore, we can define the derivative

$$
\frac{d}{d y} A\left(u, Y^{s, y}(u), F(u)\right)=A_{y}\left(u, Y^{s, y}(u), F(u)\right) \frac{Y^{s, y}(u)}{y}, \quad \text { a.s. }
$$

for $u \in[s, t+h]$. Analogously, we can define $\frac{d}{d y} D\left(u, Y^{s, y}(u), F(u)\right)$. By the mean value theorem and the growth conditions for $\varphi^{i}$, we have the estimate

$$
\begin{aligned}
& \left|\nabla \varphi_{\alpha}^{i}\right| \\
& \quad \leq K\left(1+\left|Y^{s, y+\alpha}(t+h)\right|^{p}+\left|Y^{s, y}(t+h)\right|^{p}\right) \frac{\sup _{u \in[s, t+h]}\left|Y^{s, y+\alpha}(u)-Y^{s, y}(u)\right|}{\alpha} .
\end{aligned}
$$


By the moment estimates from Step 1, the sequence $\left(\nabla \varphi_{\alpha}^{i}\right)_{\alpha}$ is uniformly integrable, since $\sup _{\alpha \in[0,1)} \mathbb{E}^{\hat{\mathbb{Q}}}\left[\left|\nabla \varphi_{\alpha}^{i}\right|^{2}\right] \leq K$. Hence, we can take the limit $\alpha \rightarrow 0$ under the expected value $\mathbb{E}^{\hat{\mathbb{Q}}}\left[\nabla \varphi_{\alpha}^{i}\right]$.

Collecting all the results, we conclude that

$$
\begin{aligned}
\theta^{*}(t) & =\theta^{*, k}(s, y, f) \\
= & \mathbb{E}_{s, y, f, k}^{\hat{Q}}\left[\int_{s}^{t+h} e^{-r(u-s)} A_{y}(u, Y(u), F(u)) Y(u)(n-N(u)) d u\right. \\
& +\int_{s}^{t+h} D_{y}(u, Y(u), F(u)) Y(u) d N(u) \\
& \left.+e^{-r(t+h-s)} \varphi_{y}^{J(t+h)}(t+h, Y(t+h), F(t+h)) Y(t+h)\right] \\
& +\mathbb{E}_{s, y, f, k}^{\hat{Q}}\left[\int_{s}^{t+h} e^{-r(u-s)} A_{f}(u, Y(u), F(u)) F(u)(n-N(u)) d u\right. \\
& +\int_{s}^{t+h} D_{f}(u, Y(u), F(u)) F(u) d N(u) \\
& \left.+e^{-r(t+h-s)} \varphi_{f}^{J(t+h)}(t+h, Y(t+h), F(t+h)) F(t+h)\right] \frac{\sigma_{F}}{\sigma_{Y}} \rho,
\end{aligned}
$$

where we use the assumption that $\left(z_{1}, z_{2}\right) \mapsto \varphi^{i}\left(t+h, z_{1}, z_{2}\right)$ is continuously differentiable for $t+h<T$.

- Step 4: Let $\vartheta^{*, k}(s, y, f)=\varphi_{y}^{k}(s, y, f) y+\varphi_{f}^{k}(s, y, f) f \frac{\sigma_{F}}{\sigma_{Y}} \rho$. We will derive estimates for $\theta^{*, k}(s, y, f), \vartheta^{*, k}(t, y, f),\left|\theta^{*, k}(s, y, f)-\vartheta^{*, k}(s, y, f)\right|$. We will only focus on the terms in $\theta^{*}$ and $\vartheta^{*}$ which contain derivatives with respect to $y$. The derivatives with respect to $f$ can be treated in the same way. From the growth condition for $\varphi^{k}$ we deduce the first estimate

$$
\begin{aligned}
& \left|\vartheta^{*, k}(s, y, f)\right| \leq K\left(1+|y|^{p}+|f|^{p}\right)(|y|+|f|) \\
& \quad \leq K\left(1+\max \left\{|y|^{p+1},|f|^{p+1}\right\}\right) \leq K\left(1+|y|^{p+1}+|f|^{p+1}\right) .
\end{aligned}
$$

Since $A$ and $D$ are Lipschitz, their derivatives are bounded. Hence, we can 
conclude that

$$
\begin{aligned}
\mid \int_{s}^{t+h} & e^{-r(u-s)} A_{y}(u, Y(u), F(u)) Y(u)(n-N(u)) d u \\
& +\int_{s}^{t+h} D_{y}(u, Y(u), F(u)) Y(u) d N(u) \\
& +e^{-r(t+h-s)} \varphi_{y}^{J(t+h)}(t+h, Y(t+h), F(t+h)) Y(t+h) \mid \\
\leq & K\left(\sup _{u \in[s, t+h]}|Y(u)| h+\sup _{u \in[s, t+h]}|Y(u)||J(t+h)-J(s)|\right. \\
& \left.+1+\sup _{u \in[s, t+h]}|Y(u)|^{p+1}+\sup _{u \in[s, t+h]}|F(u)|^{p+1}\right), \\
\leq \quad & K\left(1+\sup _{u \in[s, t+h]}|Y(u)|^{p+1}+\sup _{u \in[s, t+h]}|F(u)|^{p+1}\right) \\
& \cdot(1+h+|J(t+h)-J(s)|),
\end{aligned}
$$

where again we use the growth condition for $\varphi^{k}$ and estimates similar to (7.3). By (7.2), (7.4), the moment estimates from Step 1) and the independence between $(Y, F)$ and $N$, we get the second estimate

$$
\left|\theta^{*, k}(s, y, f)\right| \leq K\left(1+|y|^{p+1}+|f|^{p+1}\right)(1+h) .
$$

Let us introduce the following stopping times:

$$
\begin{aligned}
& \mathcal{T}_{1}=\inf \left\{s \geq t:\left|Y^{t, y}(s)-y\right| \geq \epsilon\right\} \\
& \mathcal{T}_{2}=\inf \left\{s \geq t:\left|F^{t, f}(s)-y\right| \geq \epsilon\right\} \\
& \mathcal{T}_{3}=\inf \left\{s \geq t:\left|J^{t, k}(s)-y\right| \geq 1\right\} \\
& \mathcal{T}=\mathcal{T}_{1} \wedge \mathcal{T}_{2} \wedge \mathcal{T}_{3},
\end{aligned}
$$

where we fix sufficiently small $\epsilon<1$ such that $y-\epsilon>0, f-\epsilon>0$ for the pair $(y, f)$ investigated in (4.6). Consequently, $\epsilon$ depends on $(y, f)$. By Chebyshev's inequality and the moment estimates from Step 1, we deduce 
that

$$
\begin{aligned}
\mathbb{P}(\mathcal{T} \leq & t+h) \leq \mathbb{P}\left(\sup _{s \in[t, t+h]}\left|Y^{t, y}(s)-y\right| \geq \epsilon\right) \\
& +\mathbb{P}\left(\sup _{s \in[t, t+h]}\left|F^{t, f}(s)-y\right| \geq \epsilon\right)+\mathbb{P}\left(\sup _{s \in[t, t+h]}\left|J^{t, k}(s)-k\right| \geq 1\right) \\
\leq & \frac{\mathbb{E}\left[\sup _{s \in[t, t+h]}\left|Y^{t, y}(s)-y\right|^{2}\right]}{\epsilon^{2}}+\frac{\mathbb{E}\left[\sup _{s \in[t, t+h]}\left|F^{t, f}(s)-y\right|^{2}\right]}{\epsilon^{2}} \\
& +\mathbb{E}\left[\sup _{s \in[t, t+h]}\left|J^{t, k}(s)-k\right|^{2}\right] \leq K_{y, f} h,
\end{aligned}
$$

where $K_{y, f}$ denotes a constant which depends on the pair $(y, f)$.

We now investigate the key term which we will use in the proof of the convergence of (4.6). We notice that

$$
\begin{aligned}
\mathbb{E}_{t, y, f, k} & {\left[\int_{t}^{t+h}\left|\theta^{*, J(s)}(s, Y(s), F(s))-\vartheta^{*, J(s)}(s, Y(s), F(s))\right| d s\right] } \\
\leq & \mathbb{E}_{t, y, f, k}\left[\int_{t}^{t+h} \mid \int_{s}^{t+h} e^{-r(u-s)} A_{y}(u, Y(u), F(u)) Y(u)(n-N(u)) d u\right. \\
& +\int_{s}^{t+h} D_{y}(u, Y(u), F(u)) Y(u) d N(u) \mid d s \\
& +\int_{t}^{t+h} e^{-r(t+h-s)} \mid \varphi_{y}^{J(t+h)}(t+h, Y(t+h), F(t+h)) Y(t+h) \\
& \left.\quad-\varphi_{y}^{J(s)}(s, Y(s), F(s)) Y(s) \mid d s\right] \\
& + \text { the terms with } f-\text { derivatives } \\
= & \mathbb{E}_{t, y, f, k}[H \mathbf{1}\{\mathcal{T} \leq t+h\}+H \mathbf{1}\{\mathcal{T}>t+h\}]
\end{aligned}
$$

where we use the optimal hedging strategy (7.2), Fubini's theorem and the property of conditional expectations. By (7.4), the growth condition for $\varphi^{k}$, 
the moment estimates from Step 1 and (7.6), we derive

$$
\begin{aligned}
& \mathbb{E}_{t, y, f, k}[H \mathbf{1}\{\mathcal{T} \leq t+h\}] \\
& \leq K \mathbb{E}_{t, y, f, k}\left[\left(1+\sup _{u \in[t, t+h]}|Y(u)|^{p+1}+\sup _{u \in[t, t+h]}|F(u)|^{p+1}\right) \mathbf{1}\{\mathcal{T} \leq t+h\}\right] h \\
& \leq K \sqrt{\mathbb{E}_{t, y, f, k}\left[1+\sup _{u \in[t, t+h]}|Y(u)|^{2 p+2}+\sup _{u \in[t, t+h]}|F(u)|^{2 p+2}\right]} \\
& \quad \cdot \sqrt{\mathbb{P}(\mathcal{T} \leq t+h) h} \\
& \leq K_{y, f}\left(1+|y|^{2 p+2}+|f|^{2 p+2}\right) \sqrt{h} h=K_{y, f} h^{3 / 2} .
\end{aligned}
$$

If $\mathcal{T}>t+h$, then $J(s)=k$ (or equivalently $d N(s)=0$ ), $Y(s) \in[y-\epsilon, y+$ $\epsilon], F(s) \in[f-\epsilon, f+\epsilon]$ for $s \in[t, t+h]$. Since $\varphi^{k}$ is continuously differentiable in $[0, T) \times(0, \infty) \times(0, \infty)$ by our assumption, then we can conclude that the derivatives of the function $\varphi_{y}^{k}(t, y, f)$ with respect to $t, y$ and $f$ are bounded on $[t, t+h] \times[y-\epsilon, y+\epsilon] \times[f-\epsilon, f+\epsilon]$ by a constant $K_{t, y, f}$ which depends on $(t, y, f)$. We point out that this constant is not affected when we take the limit $h \rightarrow 0$. To be more precise, first we fix $h_{0}$ such that $t+h_{0}<T$, next we find a global constant $K_{t, y, f}$ on $\left[t, t+h_{0}\right] \times[y-\epsilon, y+\epsilon] \times[f-\epsilon, f+\epsilon]$, and finally we take the limit $h \rightarrow 0, h \leq h_{0}$ in $(4.6)$. By the mean value theorem 
and the growth conditions for $\varphi^{k}$, we can conclude that

$$
\begin{aligned}
&\left|\varphi_{y}^{J(t+h)}(t+h, Y(t+h), F(t+h)) Y(t+h)-\varphi_{y}^{J(s)}(s, Y(s), F(s)) Y(s)\right| \mathbf{1}\{\mathcal{T}>t+h\} \\
& \leq \quad\left\{\left|\varphi_{y}^{k}(t+h, Y(t+h), F(t+h)) Y(t+h)-\varphi_{y}^{k}(t+h, Y(t+h), F(t+h)) Y(s)\right|\right. \\
&+\left|\varphi_{y}^{k}(t+h, Y(t+h), F(t+h)) Y(s)-\varphi_{y}^{k}(s, Y(t+h), F(t+h)) Y(s)\right| \\
&+\left|\varphi_{y}^{k}(s, Y(t+h), F(t+h)) Y(s)-\varphi_{y}^{k}(s, Y(s), F(t+h)) Y(s)\right| \\
&\left.+\left|\varphi_{y}^{k}(s, Y(s), F(t+k)) Y(s)-\varphi_{y}^{k}(Y(s), Y(s), F(s)) Y(s)\right|\right\} \mathbf{1}\{\mathcal{T}>t+h\} \\
& \leq \quad\left\{K\left(1+|Y(t+h)|^{p}+|F(t+h)|^{p}\right)|Y(t+h)-Y(s)|\right. \\
&+K_{t, y, f} Y(s) h \\
&\left.+K_{t, y, f}|Y(t+h)-Y(s)| Y(s)+K_{t, y, f}|F(t+h)-F(s)| Y(s)\right\} \mathbf{1}\{\mathcal{T}>t+h\} \\
& \leq \quad K_{t, y, f}\left(1+\sup _{u \in[t, t+h]}|Y(u)|^{p}+\sup _{u \in[t, t+h]}|F(u)|^{p}\right) \\
& \cdot\left(h+\sup _{u \in[t, t+h]}|Y(t+h)-Y(u)|+\sup _{u \in[t, t+h]}|F(t+h)-F(u)|\right) \\
& \leq K_{t, y, f}\left(1+\sup _{u \in[t, t+h]}|Y(u)|^{p}+\sup _{u \in[t, t+h]}|F(u)|^{p}\right) \\
& \cdot\left(h+\sup _{u \in[t, t+h]}|Y(u)-Y(t)|+\sup _{u \in[t, t+h]}|F(u)-F(t)|\right) .
\end{aligned}
$$

Combining the above estimate with

$$
\begin{aligned}
& \mid \int_{s}^{t+h} e^{-r(u-s)} A_{y}(u, Y(u), F(u)) Y(u)(n-N(u)) d u \\
& \quad+\int_{s}^{t+h} D_{y}(u, Y(u), F(u)) Y(u) d N(u) \mid \mathbf{1}\{\mathcal{T}>t+h\} \\
& \quad \leq \quad K \sup _{u \in[t, t+h]}|Y(u)| h,
\end{aligned}
$$

and using the moment estimates from Step 1, we find

$$
\mathbb{E}_{t, y, f, k}[H \mathbf{1}\{\mathcal{T}>t+h\}] \leq K_{t, y, f} h^{3 / 2} .
$$

Collecting (7.7), (7.8) and (7.9), we establish the convergence

$$
\lim _{h \rightarrow 0} \mathbb{E}_{t, y, f, k}\left[\frac{1}{h} \int_{t}^{t+h}\left|\theta^{*, J(s)}(s, Y(s), F(s))-\vartheta^{*, J(s)}(s, Y(s), F(s))\right| d s\right]=0 .
$$


By the dominated convergence theorem (justified by (7.3) and the moment estimates from Step 1) and the differentiability of the Lebesgue integral, we find that

$$
\begin{gathered}
\lim _{h \rightarrow 0} \mathbb{E}_{t, y, f, k}\left[\frac{1}{h} \int_{t}^{t+h} \vartheta^{*, J(s)}(s, Y(s), F(s)) d s\right] \\
=\varphi_{y}^{k}(t, y, f) y+\varphi_{f}^{k}(t, y, f) f \frac{\sigma_{F}}{\sigma_{Y}} \rho .
\end{gathered}
$$

- Step 5: We can now take the limits of the terms on the left hand side of equation (4.6). We divide the equation (4.6) by $h$. We deal with the first term in (4.6). The optimal hedging strategy $\theta_{B(t, t+h), \varphi(t+h)}^{*}$ is given by (7.2), and the dynamics of the hedging portfolio is given by (3.4). Since $\varphi^{k} \in \mathcal{C}^{1,2,2}([t, t+h] \times(0, \infty) \times(0, \infty))$ by our assumption, then we can apply Itô's formula to derive the dynamics of $X^{[t, t+h]}$. The dynamics of $X^{[t, t+h]}$ on $[t, t+h]$ is given by (5.2). We have

$$
\begin{aligned}
& \frac{\mathbb{E}_{t, y, f, k}\left[X^{[t, t+h]}(t+h) e^{-r h}-X^{[t, t+h]}(t)\right]}{h} \\
& \quad=\frac{\mathbb{E}_{t, y, f, k}\left[X^{[t, t+h]}(t+h) e^{-r(t+h)}-X^{[t, t+h]}(t) e^{-r t}\right]}{h} e^{r t} \\
& \quad=\frac{\mathbb{E}_{t, y, f, k}\left[\int_{t}^{t+h}\left(-r e^{-r s} X^{[t, t+h]}(s) d s+e^{-r s} d X^{[t, t+h]}(s)\right]\right.}{h} e^{r t} .
\end{aligned}
$$

From the proof of Proposition 3.1 we can conclude that the optimal hedging strategy $\theta_{B(t, t+h), \varphi(t+h)}^{*}$ is square integrable on $[t, t+h]$. This square integrability, together with the growth conditions for $D, \varphi^{k}$ and the moment estimates from Step 1, allows us to conclude that the three stochastic integrals in (5.2) are martingales with expected values equal to zero. We can write $(7.12)$ as

$$
\begin{gathered}
\frac{\mathbb{E}_{t, y, f, k}\left[-\int_{t}^{t+h} e^{-r s} \theta^{*, J(s)}(s, Y(s), F(s))\left(\mu_{Y}-r\right) d s\right]}{h} e^{r t} \\
+\frac{\mathbb{E}_{t, y, f, k}\left[\int_{t}^{t+h} e^{-r s} \Psi^{J(s)}(s, Y(s), F(s)) d s\right]}{h} e^{r t},
\end{gathered}
$$

where $\Psi$ collects all the remaining terms. We use (7.10) and (7.11), and we conclude that the limit $h \rightarrow 0$ of the first term in $(7.13)$ is equal to 
$-\vartheta^{*, k}(t, y, f)\left(\mu_{Y}-r\right)$. Since the function $\varphi^{k}$ satisfies the PDE (5.8), we have

$$
\begin{aligned}
& \Psi^{k}(s, y, f)=\varphi_{y}^{k}(s, y, f) y(\mu-r)+\varphi_{f}^{k}(s, y, f) f \frac{\mu_{Y}-r}{\sigma_{Y}} \sigma_{F} \rho \\
& \quad-\Phi^{k}\left(\varphi_{f}^{k}(s, y, f) f \sigma_{F} \sqrt{1-\rho^{2}}, \varphi^{k-1}(s, y, f)+D(s, y, f)-\varphi^{k}(s, y, f)\right),
\end{aligned}
$$

and, using the growth condition for $\varphi^{k}$, we can derive the estimate

$$
\left|\Psi^{k}(s, y, f)\right| \leq K\left(1+|y|^{2 p+2}+|f|^{2 p+2}\right),
$$

By the dominated convergence theorem (justified by (7.14) and the moment estimates from Step 1) and the differentiability of the Lebesgue integral, we can derive that the limit for $h \rightarrow 0$ of the second term in (7.13) is equal to $\Psi^{k}(t, y, f)$. The limit (5.5) is proved.

- Step 6: We now deal with the second term in (4.6). It is clear that

$$
\operatorname{Var}[\xi]=\left(E\left[\left(\xi e^{-r t}\right)^{2}\right]-\left(\mathbb{E}\left[\xi e^{-r t}\right]\right)^{2}\right) e^{2 r t} .
$$

We start with the second term in (7.14). Using the dynamics (5.2), equations (7.12)-(7.13) and applying the Burkholder-Davis-Gundy inequality, we can 
derive

$$
\begin{aligned}
\mathbb{E}_{t, y, f, k} & {\left[\sup _{s \in[t, t+h]}\left|X^{[t, t+h]}(s) e^{-r s}-X^{[t, t+h]}(t) e^{-r t}\right|^{q}\right] } \\
= & \mathbb{E}_{t, y, f, k}\left[\sup _{s \in[t, t+h]} \mid-\int_{t}^{s} e^{-r u} \theta^{*, J(u-)}(u, Y(u), F(u))\left(\mu_{Y}-r\right) d u\right. \\
& +\int_{t}^{s} e^{-r u} \Psi^{J(u-)}(u, Y(u), F(u)) d u \\
& +\int_{t}^{s} e^{-r u}\left(\theta^{*, J(u-)}(u, Y(u), F(u))-\vartheta^{*, J(u-)}(u, Y(u), F(u))\right) \sigma_{Y} d W_{1}(u) \\
& +\int_{t}^{s} e^{-r u} \varphi_{f}^{J(u-)}(u, Y(u), F(u)) F(u) \sigma_{F} \sqrt{1-\rho^{2}} d W_{2}(u), \\
& +\int_{t}^{s} e^{-r u}\left(\varphi^{J(u-)-1}(u, Y(u), F(u))+D(u, Y(u), F(u))\right. \\
& \left.\left.-\varphi^{J(u-)}(u, Y(u), F(u))\right)\left.d \tilde{N}(u)\right|^{q}\right] \\
& K \mathbb{E}_{t, y, f, k}\left[h \int_{t}^{t+h}\left|\theta^{*, J(u)}(u, Y(u), F(u))\right|^{q} d u\right. \\
+ & h \int_{t}^{t+h}\left|\Psi^{J(u)}(u, Y(u), F(u))\right|^{q} d u \\
+ & \left|\int_{t}^{t+h}\right| \theta^{*, J(u)}(u, Y(u), F(u))-\left.\left.\vartheta^{*, J(u)}(u, Y(u), F(u))\right|^{2} d u\right|^{q / 2} \\
+ & \left.\left.\left|\int_{t}^{t+h}\right| \varphi_{f}^{J(u)}(u, Y(u), F(u)) F(u)\right|^{2} d u\right|^{q / 2} \\
+ & \left|\int_{t}^{t+h}\right| \varphi^{J(u)-1}(u, Y(u), F(u))+D(u, Y(u), F(u)) \\
& \left.-\left.\left.\varphi^{J(u)}(u, Y(u), F(u))\right|^{2} d N(u)\right|^{q / 2}\right], \\
& \\
& \\
&
\end{aligned}
$$

for any $q \geq 2$. By the growth conditions for $A, D, \varphi^{k}, \Psi^{k}$, the moment estimates from Step 1) and the estimates (7.3), (7.5), we can conclude that

$$
\mathbb{E}_{t, y, f, k}\left[\sup _{s \in[t, t+h]}\left|X^{[t, t+h]}(s) e^{-r s}-X^{[t, t+h]}(t) e^{-r t}\right|^{q}\right] \leq K_{y, f} h, \quad q \geq 2 .
$$

The estimate 7.15 and the limit 5.5 now yield

$$
\lim _{h \rightarrow 0} \frac{\left(\mathbb{E}_{t, y, f, k}\left[X^{[t, t+h]}(t+h) e^{-r(t+h)}-X^{[t, t+h]}(t) e^{-r t}\right]\right)^{2}}{h} e^{2 r t}=0 .
$$


We investigate the first term in 7.14):

$$
\begin{aligned}
\mathbb{E}_{t, y, f, k} & {\left[\left|X^{[t, t+h]}(t+h) e^{-r(t+h)}-X^{[t, t+h]}(t) e^{-r t}\right|^{2}\right] } \\
= & \mathbb{E}_{t, y, f, k}\left[\left|X^{[t, t+h]}(t+h) e^{-r(t+h)}\right|^{2}\right. \\
- & \left.-2 X^{[t, t+h]}(t+h) e^{-r(t+h)} X^{[t, t+h]}(t) e^{-r t}+\left|X^{[t, t+h]}(t) e^{-r t}\right|^{2}\right] \\
= & 2 \mathbb{E}_{t, y, f, k}\left[\int_{t}^{t+h}\left(X^{[t, t+h]}(s) e^{-r s}-X^{[t, t+h]}(t) e^{-r t}\right)\right. \\
& \left.\cdot\left(-r e^{-r s} X^{[t, t+h]}(s) d s+e^{-r s} d X^{[t, t+h]}(s)\right)\right] \\
+ & \mathbb{E}_{t, y, f, k}\left[\int_{t}^{t+h} e^{-2 r s} d\left[X^{[t, t+h]}, X^{t, t+h]}\right](s)\right]:=2 E_{1}^{h}+E_{2}^{h},
\end{aligned}
$$

where we apply Itô formula to $\left|X^{[t, t+h]}(s) e^{-r s}\right|^{2}$ and use the dynamics of the discounted process $X^{[t, t+h]}(s) e^{-r s}$.

First, we consider $E_{1}^{h}$. By (7.5) and the moment estimates from Step 1 ), we can prove that $\mathbb{E}_{t, y, f, k}\left[\int_{t}^{t+h}\left|\theta^{*, J(s)}(s, Y(s), F(s))\right|^{q} d s\right]<\infty$ for any $q \geq 2$. This integrability condition together with the growth conditions for $D, \varphi^{k}$ and estimate (7.15) yield that the stochastic integrals with respect to Brownian motions and the compensated counting process which we have in $E_{1}^{h}$, see (5.2), are square integrable martingales and their expected values are equal to zero. Using (7.13) we have

$$
\begin{aligned}
\left|E_{1}^{h}\right| \leq & \sqrt{\mathbb{E}_{t, y, f, k}\left[\sup _{s \in[t, t+h]}\left|X^{[t, t+h]}(s) e^{-r s}-X^{[t, t+h]}(t) e^{-r t}\right|^{2}\right]} \\
& \cdot \sqrt{\mathbb{E}_{t, y, f, k}\left[\sup _{s \in[t, t+h]}\left|\theta^{*, J(s)}(s, Y(s), F(s))\right|^{2}+\sup _{s \in[t, t+h]}\left|\Psi^{J(s)}(s, Y(s), F(s))\right|^{2}\right]} h \\
\leq & K_{y, f} \sqrt{h} h,
\end{aligned}
$$

where in the last line we use the estimates $(7.5),(7.15),(7.14)$ and the moment estimates from Step 1). Hence, we conclude that

$$
\lim _{h \rightarrow 0} \frac{1}{h} E_{1}^{h}=0 .
$$

We finally deal with $E_{2}^{h}$. We can calculate the quadratic variation and we 
find that

$$
\begin{aligned}
E_{2}^{h}= & \mathbb{E}_{t, y, f, k}\left[\int _ { t } ^ { t + h } e ^ { - 2 r s } \left(\left|\theta^{*, J(s)}(s, Y(s), F(s))-\vartheta^{*, J(s)}(s, Y(s), F(s))\right|^{2}\right.\right. \\
+ & \left|\varphi_{f}^{J(s)}(s, Y(s), F(s))\right|^{2} F^{2}(s) \sigma_{F}^{2}\left(1-\rho^{2}\right) \\
+ & \mid \varphi^{J(s)-1}(s, Y(s), F(s))+D(s, Y(s), F(s)) \\
& \left.\left.-\left.\varphi^{J(s)}(s, Y(s), F(s))\right|^{2}(n-N(s)) \lambda(s)\right) d s\right] .
\end{aligned}
$$

As in (7.10), we can deduce that

$\lim _{h \rightarrow 0} \mathbb{E}_{t, y, f, k}\left[\frac{1}{h} \int_{t}^{t+h}\left|\theta^{*, J(s)}(s, Y(s), F(s))-\vartheta^{*, J(s)}(s, Y(s), F(s))\right|^{2} d s\right]=0$.

By the dominated convergence theorem and the differentiability of the Lebesgue integral, we derive the limit:

$$
\begin{aligned}
\lim _{h \rightarrow 0} \frac{1}{h} E_{2}^{h}= & e^{-2 r t}\left(\left(\varphi_{f}^{k}(t, y, f)\right)^{2} f^{2} \sigma_{F}^{2}\left(1-\rho^{2}\right)\right. \\
& \left.+\left(\varphi^{k-1}(t, y, f)+D(t, y, f)-\varphi^{k}(t, y, f)\right)^{2} k \lambda(t)\right) .
\end{aligned}
$$

We collect (7.14), 7.16)- 7.19) and we get the desired limit (5.6).

Proof of Theorem 5.2; (i) The representation (5.9) is a Feynman-Kac formula for the PDEs (5.8). The dynamics of $(Y, F, J)$ under $\hat{\mathbb{Q}}$ are given by 2.16-2.18). By the growth conditions for $A, D, S, \varphi^{k}, \Phi^{k}$ and the moment estimates from Step 1) in the proof of Theorem 5.1, we can show that the random variable inside the expected value in $(5.9)$ has finite moments of all orders under $\hat{\mathbb{Q}}$. Consequently, the process $\varphi^{J(t)}(t, Y(t), F(t)) e^{-r t}+$ $\int_{0}^{t} e^{-r s} d B(s)+\int_{0}^{t} e^{-r s} \Phi(s) d s$ is a $\hat{\mathbb{Q}}$-martingale. Since $\varphi^{k}$ is smooth, we can apply Itô's formula and the drift term of the martingale under $\hat{\mathbb{Q}}$ must be zero, see also the proof of Proposition 3.1.

(ii) By our assumption, the risk premiums $\zeta_{V}, \chi_{V}, \zeta_{S D}, \chi_{S D}$ satisfy 2.15) and, consequently, we can define the equivalent probability measure $\frac{d \tilde{\mathbb{Q}}}{d \hat{\mathbb{Q}}} \mid \mathcal{F}_{T}=$ $\mathcal{M}^{\zeta, \chi}(T)$ for $(\zeta, \chi) \in\left\{\left(\zeta_{V}, \chi_{V}\right),\left(\zeta_{S D}, \chi_{S D}\right)\right\}$. The dynamics of $(Y, F, J)$ under $\tilde{\mathbb{Q}}$ are given by 2.17$)-(2.18)$. Since $\mathbb{E}^{\hat{\mathbb{Q}}}\left[\left|\mathcal{M}^{\zeta, \chi}(T)\right|^{l}\right]<\infty$ for some $l>1$ and 
$B(T)$ has finite moments of all orders under $\hat{\mathbb{Q}}$, the random variable inside the expected value in $(5.10)$ has finite moments of all orders under $\tilde{\mathbb{Q}}$. We next proceed as in point (i) and we check that

$$
\begin{aligned}
& \Phi^{k}\left(\varphi_{f}^{k}(t, y, f) f \sigma_{F} \sqrt{1-\rho^{2}}, \varphi^{k-1}(t, y, f)+D(t, y, f)-\varphi^{k}(t, y, f)\right) \\
&= \frac{1}{2} \gamma\left(\varphi_{f}^{k}(t, y, f) f \sigma_{F} \sqrt{1-\rho^{2}} \zeta(t)\right. \\
&\left.\quad+\left(\varphi^{k-1}(t, y, f)+D(t, y, f)-\varphi^{k}(t, y, f)\right) k \lambda(t) \chi(t)\right) .
\end{aligned}
$$

We can also deduce that (A4) is satisfied, see point (iii).

(iii) Let us consider $\tilde{B}(t)=B^{Y}(t)+B(t)$ where $B^{Y}$ is a hedgeable process and $B$ is an arbitrary process. Let $\tilde{\varphi}$ denotes the valuation operator which satisfies the PDEs (5.8) for the benefit stream $\tilde{B}$. Similarly, $\varphi^{Y}$ and $\varphi$ are the valuation operators for $B^{Y}$ and $B$, respectively. Since $B^{Y}$ only depends on $Y$, we expect that $\varphi^{Y, k}(t, y, f)=\varphi^{Y}(t, y)$ and the PDEs (5.8) reduce to

$$
\begin{aligned}
& \varphi_{t}^{Y}(t, y)+\varphi_{y}^{Y}(t, y) y r+\frac{1}{2} \varphi_{y y}^{Y}(t, y) y^{2} \sigma_{Y}^{2}+A(t, y)-\varphi^{Y}(t, y) r=0, \\
& \varphi(T, y)=S(y) .
\end{aligned}
$$

By Theorem 1 in Heath \& Schweizer (2000) there exists a unique solution to (7.20) with the representation

$$
\varphi^{Y}(t, y)=\mathbb{E}_{t, y}^{\hat{\mathbb{Q}}}\left[\int_{t}^{T} e^{-r(s-t)} d B^{Y}(s)\right] .
$$

By direct substitution and additivity of derivatives, we can also show that the functions $\psi^{k}(t, y, f)=\varphi^{Y}(t, y)+\varphi^{k}(t, y, f)$ satisfy the PDEs (5.8) for $\tilde{B}$. Consequently, $\psi^{k}(t, y, f)=\tilde{\varphi}^{k}(t, y, f)$ and the valuation operator is marketconsistent.

For an orthogonal process $B^{N}$ we expect that $\varphi^{B^{N}, k}(t, y, f)=\varphi^{B^{N}, k}(t)$. The system of PDEs (5.8) reduces to

$$
\begin{aligned}
& \varphi_{t}^{k}(t)+k A(t)+\left(\varphi^{k-1}(t)+D(t)-\varphi^{k}(t)\right) k \lambda(t)-\varphi^{k}(t) r \\
& +\Phi^{k}\left(t, 0, \varphi^{k-1}(t)+D(t)-\varphi^{k}(t)\right)=0 \\
& \varphi^{k}(T)=k S .
\end{aligned}
$$


Clearly, $\varphi^{0}(t)=0$. If the PDEs 7.21 have solutions $\left(\varphi^{k}\right)_{k=1, \ldots, n}$, which are derived recursively, then we can conclude that $\varphi^{k}$ indeed only depends on $(t, k)$ and the future claims $A, D, S$ from the benefit stream $B^{N}$. We now use (5.9). For the standard deviation risk margin we have

$$
\begin{aligned}
\varphi^{k}(t)= & \mathbb{E}_{t, k}^{\mathbb{P}}\left[\int_{t}^{T} e^{-r(s-t)} d B^{N}(s)\right. \\
& \left.+\frac{1}{2} \gamma \int_{t}^{T} e^{-r(s-t)}\left|\varphi^{J(s-)-1}(s)+D(s)-\varphi^{J(s-)}(s)\right| \sqrt{J(s-) \lambda(s)} d s\right] \\
= & \mathbb{E}_{t, k}^{\mathbb{P}}\left[\int_{t}^{T} e^{-r(s-t)} d B^{N}(s)\right]+R M_{B^{N}(t, T]}^{a c t}(t),
\end{aligned}
$$

and for the variance risk margin we find

$$
\begin{aligned}
\varphi^{k}(t)= & \mathbb{E}_{t, k}^{\mathbb{P}}\left[\int_{t}^{T} e^{-r(s-t)} d B^{N}(s)\right. \\
& \left.+\frac{1}{2} \gamma \int_{t}^{T} e^{-r(s-t)}\left|\varphi^{J(s-)-1}(s)+D(s)-\varphi^{J(s-)}(s)\right|^{2} J(s-) \lambda(s) d s\right] \\
= & \mathbb{E}_{t, k}^{\mathbb{P}}\left[\int_{t}^{T} e^{-r(s-t)} d B^{N}(s)\right]+R M_{B^{N}(t, T]}^{a c t}(t),
\end{aligned}
$$

where in both cases the risk margin valuation operator $R M_{B^{N}(t, T]}^{a c t}$ is actuarial. More precisely, it takes the form (2.11) and the valuation operator is actuarial since (2.9) holds.

Proof of Theorem 5.3: The formula for $\vartheta^{*}$ is deduced from 7.10 and (7.11). Using the properties of the valuation operator $\varphi$ discussed in the proof of Theorem 5.2, we can easily prove that our hedging strategy $\vartheta^{*}$ is market-consistent and actuarial.

\section{References}

Albrecher, H., Bauer, D., Embrechts, P., Filipović, D., Koch-Medina, P., Korn, R., Loisel, S., Pelsser, A., Schiller, F., Schmeiser, H. et al. (2018), 'Asset-liability management for long-term insurance business', European Actuarial Journal 8(1), 9-25. 
Barigou, K., Chen, Z. \& Dhaene, J. (2018), 'Fair valuation of insurance liabilities: Merging actuarial judgement with market- and time-consistency'. Working paper.

Barigou, K. \& Dhaene, J. (2018), 'Fair valuation of insurance liabilities via mean-variance hedging in a multi-period setting', Scandinavian Actuarial Journal .

URL: https://doi.org/10.1080/03461238.2018.1528477

Barndorff-Nielsen, O. E., Graversen, S. E., Jacod, J., Podolskij, M. \& Shephard, N. (2006), A central limit theorem for realised power and bipower variations of continuous semimartingales, in 'From Stochastic Calculus to Mathematical Finance', Springer, pp. 33-68.

Barrieu, P. \& Karoui, N. E. (2005), Pricing, hedging and optimally designing derivatives via minimization of risk measures, in 'Indifference Pricing: Theory and Applications', Princeton University Press, pp. 77-141.

Black, F. \& Scholes, M. (1973), 'The pricing of options and corporate liabilities', Journal of Political Economy 81(3), 637-654.

Černỳ, A. \& Kallsen, J. (2009), 'Hedging by sequential regressions revisited', Mathematical Finance 19(4), 591-617.

Cheridito, P., Delbaen, F. \& Kupper, M. (2006), 'Dynamic monetary risk measures for bounded discrete-time processes', Electronic Journal of Probability 11, 57-106.

Cont, R. \& Voltchkova, E. (2005), 'Integro-differential equations for option prices in exponential lévy models', Finance and Stochastics 9(3), 299-325.

Delbaen, F. \& Schachermayer, W. (2006), The Mathematics of Arbitrage, Springer Science \& Business Media.

Delong, Ł. (2013), Backward Stochastic Differential Equations with Jumps and Their Actuarial and Financial Applications, Springer. 
Delong, Ł., Dhaene, J. \& Barigou, K. (2018), 'Fair valuation of insurance liability cash-flow streams in continuous time: Applications'. Submitted.

Dhaene, J., Stassen, B., Barigou, K., Linders, D. \& Chen, Z. (2017), 'Fair valuation of insurance liabilities: merging actuarial judgement and marketconsistency', Insurance: Mathematics and Economics 76, 14-27.

El Karoui, N., Peng, S. \& Quenez, M. C. (1997), 'Backward stochastic differential equations in finance', Mathematical Finance 7(1), 1-71.

Föllmer, H. \& Schweizer, M. (1988), 'Hedging by sequential regression: An introduction to the mathematics of option trading', ASTIN Bulletin: The Journal of the IAA 18(2), 147-160.

Happ, S., Merz, M. \& Wüthrich, M. V. (2015), 'Best-estimate claims reserves in incomplete markets', European Actuarial Journal 5(1), 55-77.

Heath, D. \& Schweizer, M. (2000), 'Martingales versus pdes in finance: an equivalence result with examples', Journal of Applied Probability 37(4), 947-957.

Hodges, S. D. \& Neuberger, A. (1989), 'Optimal replication of contingent claims under transaction costs', Review of Futures Markets 8(2), 222-239.

Hu, Y., Jin, H. \& Zhou, X. Y. (2012), 'Time-inconsistent stochastic linear-quadratic control', SIAM Journal on Control and Optimization 50(3), 1548-1572.

Jacod, J. (2008), 'Asymptotic properties of realized power variations and related functionals of semimartingales', Stochastic Processes and Their Applications 118(4), 517-559.

Kaas, R., Goovaerts, M., Dhaene, J. \& Denuit, M. (2008), Modern Actuarial Risk Theory: Using R, Springer Science \& Business Media. 
Kazamaki, N. (2006), Continuous Exponential Martingales and BMO, Springer.

Malamud, S., Trubowitz, E. \& Wüthrich, M. (2008), 'Market consistent pricing of insurance products', ASTIN Bulletin: The Journal of the IAA 38(2), 483-526.

Møller, T. \& Steffensen, M. (2007), Market-Valuation Methods in Life and Pension insurance, Cambridge University Press.

Morlais, M.-A. (2010), 'A new existence result for quadratic bsdes with jumps with application to the utility maximization problem', Stochastic Processes and Their Applications 120(10), 1966-1995.

Norberg, R. (2014), 'Life insurance mathematics', Wiley StatsRef: Statistics Reference Online.

Pelsser, A. (2010), 'Time-consistent and market-consistent actuarial valuations'. Available at SSRN: https://ssrn.com/abstract=1551323.

Pelsser, A. \& Ghalehjooghi, A. S. (2016), 'Time-consistent actuarial valuations', Insurance: Mathematics and Economics 66, 97-112.

Pelsser, A. \& Stadje, M. (2014), 'Time-consistent and market-consistent evaluations', Mathematical Finance 24(1), 25-65.

Rosazza Gianin, E. (2006), 'Risk measures via g-expectations', Insurance: Mathematics and Economics 39(1), 19-34.

Stadje, M. (2010), 'Extending dynamic convex risk measures from discrete time to continuous time: a convergence approach', Insurance: Mathematics and Economics 47(1), 391-404. 